\title{
Second order analysis for the optimal control of parabolic equations under control and final state constraints
}

\author{
F. J. Silva *†
}

December 18, 2014

\begin{abstract}
We consider the optimal control of a semilinear parabolic equation with pointwise bounds constraints on the control and finitely many integral constraints on the final state. Using the standard Robinson's constraint qualification [37], we provide a second order necessary condition over a set of strictly critical directions. The main feature of this result is that the qualification condition needed for the second order analysis is the same as for classical finite-dimensional problems and does not imply the uniqueness of the Lagrange multiplier. We establish also a second order sufficient optimality condition which implies, for problems with a quadratic Hamiltonian, the equivalence between solutions satisfying the quadratic growth property in the $L^{1}$ and $L^{\infty}$ topologies.
\end{abstract}

Keywords. Optimal control, parabolic equations, box constraints for the control, finitely many constraints for the state, Robinson constraint qualification, second order optimality conditions, quadratic Hamiltonian.

MSC. 49J20, 49K20, 35Q93.

\section{Introduction}

The study of second order optimality conditions for optimal control problems governed by semilinear elliptic or parabolic equations has received a considerable attention over the last two decades (see $[26,21,6,22,13,5,17,15,18,39,19,9,20,3]$ and the references therein). The main reason is that under the absence of convexity, this theory plays a crucial role for the analysis of stability properties of the problem at hand. As a consequence, second order analysis is fundamental for establishing the local convergence of the solutions of some finite dimensional discretizations as well as the convergence of some optimization methods in function spaces. We refer the reader to the recent monograph [41] for a rather complete account of the theory and to [27,31], and the references therein, for the analysis of some associated Newton-based methods.

The study of optimality conditions for optimal control problems has several difficulties depending on the type of constraints considered. A common feature is that, in contrast to finite-dimensional optimization problems where the norms are equivalent, a two-norm approach for second order optimality conditions is necessary (see $[29,36]$ ). In fact, the expansion of the cost function in terms of

\footnotetext{
*XLIM - DMI UMR CNRS 7252 Faculté des Sciences et Techniques, Université de Limoges (francisco.silva@unilim.fr) +33587506787

${ }^{\dagger}$ This research benefited from the support of the "FMJH Program Gaspar Monge in optimization and operation research", and from the support to this program from EDF.
} 
control perturbations shows that if a second order sufficient condition has to be supposed, the natural norm for the coercitivity condition of the associated quadratic form is the $L^{2}$-norm. On the other hand, since the cost function is in general $C^{2}$ with respect to the $L^{\infty}$-norm (and not with respect to the $L^{2}$-norm), the neighborhood on which local optimality can be expected is a $L^{\infty}$-neighborhood of the nominal point.

In this article we consider the optimal control problem of a semilinear parabolic equation, where bounds constraints are imposed on the control for almost all (a.a.) $(t, x)$ and a finite number of equality and inequality constraints in integral form are imposed on the final state. Since the bounds constraints on the control are polyhedric, our framework for the constraints is a particular case of the one introduced in [13] and in [18]. The main difference with respect to [13] is that in our case the cost function and the finite dimensional constraints are not $C^{2}$ in the $L^{2}$ topology. We could have considered other types of finitely many constraints for the state, but we have decided to focus in the case of final state constraints because of its analogy with the classical setting in the optimal control of ODEs.

For a weak local solution $\bar{u}$, i.e. when the cost is minimized at $\bar{u}$ locally on a neighborhood in $L^{\infty}$ relative to the feasible set (see Definition 2.4(i)), we prove two types of second order necessary conditions. The first one is stated in Theorem 5.2, which is our main result. It shows that, under the classical Robinson constraint qualification, for every direction $v$ which belongs to the strict critical cone at $\bar{u}$, there exists a Lagrange multiplier associated to the finitely-many integral constraints, such that the associated quadratic form, which depends on $\lambda$, is non-negative at $v$. The strength of this result is that only the standard Robinson condition is assumed (see [37]), in analogy to finite dimensional optimization problems. The drawback is that the strict critical cone is in general smaller than the usual critical cone. However, under a strict complementary assumption associated to the finite dimensional problem appearing in Pontryagin's principle, both cones coincide. We remark that, at least in the case of pure control constraints, the strict complementary assumption plays an important role in the asymptotic expansion of solutions under data perturbation (see [35, 12]). The proof of Theorem 5.2 is essentially based on the ideas exposed in $[10,8]$, a precise second order expansion of the Lagrangian with respect to bounded perturbations of the local solution (see Section 3), some density arguments and Dmitruk's lemma (see [23]).

The second type of second order necessary optimality condition is proved in Theorem 5.7. The main assumption of this result is a strong regularity property introduced in [40] and revisited in [13], which implies Robinson constraint qualification and also the uniqueness of the Lagrange multiplier associated to the final state constraints. The key point is that this assumption allows to approximate the critical cone by its intersection with the radial cone of the polyhedric constraints. This regularity condition has been applied in [13] in order to develop a second order theory for optimization problems with partially polyhedric constraints and $C^{2}$ data. The main advantage of the result in Theorem 5.7 is that the associated quadratic form is non-negative for all critical directions, but has the disadvantage that requires stronger assumptions than those expected from classical optimization theory. We point out that the same type of result can also be obtained by using the techniques in $[15,18]$, but under a stronger surjectivity assumption (see Remark 5.3(ii)). The problem of finding a second order necessary condition over the classical critical cone, without requiring additional assumptions besides Robinson condition, remains still open in the field of optimization of PDE systems.

Using classical results about the dependence of solutions of linear parabolic equations on the right hand side (which are recalled in Section 2) and the asymptotic expansions studied in Section 3 , the proof of the second order sufficient condition for the weak quadratic growth property, under the assumption that the associated quadratic form is a Legendre form, does not present particular difficulties. We have chosen this approach since in the case of a quadratic Hamiltonian, the Legendre form assumption is automatically verified. As a consequence, under the assumption that the strict critical cone coincides with the usual critical cone, or alternatively that the stronger form of Robinson 
condition is verified, we have the equivalence of the quadratic growth property with respect to the $L^{\infty}$ and $L^{1}$ topologies (see Section 6). Apparently, this characterization is new in the literature.

The paper is organized as follows. In Section 2 we fix some notations, we recall some basic facts about linear and semilinear parabolic equations and we state the optimal control problem under consideration together with the standard assumptions. Using the asymptotic expansions for the Lagrangian studied in Section 3, we provide in Sections 5 and 6 our main results. Namely, we prove the two types of second order necessary condition described above and also a sufficient second order optimality condition. The latter is crucial in the analysis of optimal control problems with quadratic Hamiltonians for which the equivalence between weak and $L^{1,1}$ solutions satisfying the quadratic growth condition is established. We end our paper by explaining how our assumptions have to be modified in the case of unbounded controls.

\section{Preliminaries}

Let us fix $T>0$ and recall that given a Banach space $\left(X,\|\cdot\|_{X}\right)$ and $s \in\left[1, \infty\left[\right.\right.$, the space $L^{s}([0, T] ; X)$ (respectively, $\left.L^{\infty}([0, T] ; X)\right)$ consists in all measurable functions $f:[0, T] \rightarrow X$ satisfying that

$$
\|f\|_{L^{s}([0, T] ; X)}:=\left(\int_{0}^{T}\|f(t)\|_{X}^{s} \mathrm{~d} t\right)^{\frac{1}{s}}<\infty \quad\left(\text { respectively } \quad\|f\|_{L^{\infty}([0, T] ; X)}:=\operatorname{ess} \sup _{t \in[0, T]}\|f(t)\|_{X}<\infty\right) .
$$

We have that $\left(L^{s}([0, T] ; X),\|\cdot\|_{L^{s}([0, T] ; X)}\right)$ is a Banach space (see e.g. [34]). Let us also recall that $W^{1, s}([0, T] ; X)$ is the space of all $X$-valued distributions $f \in L^{s}([0, T] ; X)$ such that the vector valued distributional derivative $\partial_{t} f$ can be identified with an element in $L^{s}([0, T] ; X)$. Endowed with the norm $\|f\|_{W^{1, s}([0, T] ; X)}:=\|f\|_{L^{s}([0, T] ; X)}+\left\|\partial_{t} f\right\|_{L^{s}([0, T] ; X)}$, we have that $W^{1, s}([0, T] ; X)$ is a Banach space.

In this article, $\Omega \subseteq \mathbb{R}^{d}$ denotes a non-empty bounded open set with a smooth boundary. Set $Q=] 0, T[\times \Omega$ and $\Sigma=] 0, T\left[\times \partial \Omega\right.$. For notational convenience, for $s_{1}, s_{2} \in[1, \infty]$, we set $L^{s_{1}, s_{2}}(Q):=$ $L^{s_{1}}\left([0, T] ; L^{s_{2}}(\Omega)\right)$ and $\|f\|_{s_{1}, s_{2}}:=\|f\|_{L^{s_{1}}\left([0, T] ; L^{s_{2}}(\Omega)\right)}$. We also write $\|\cdot\|_{s_{1}}:=\|\cdot\|_{L^{s_{1}}(\Omega)}$. Given $\alpha, \beta>0$ we recall that $C^{\alpha}(\bar{\Omega})$ is the space of $\alpha$-Hölder continuous functions in $\bar{\Omega}$ and $C^{\alpha, \beta}(\bar{Q})$ is the space of functions defined on $\bar{Q}$ which are $\alpha$-Hölder continuous with respect to $t$ and $\beta$-Hölder continuous with respect to $x$.

We will also need the following notations: for any two Banach spaces $X, Y$ paired in duality, $\langle\cdot, \cdot\rangle_{X, Y}$ denotes the associated bilinear form. We denote $\mathbb{R}_{+}:=\{r \in \mathbb{R} ; r \geq 0\}$ and $\mathbb{R}_{-}:=\{r \in$ $\mathbb{R} ; r \leq 0\}$. Given a Banach space $\left(X,\|\cdot\|_{X}\right)$ and a set $A \subseteq X$, we let $d_{X}(z, A):=\inf \left\{\|z-x\|_{X} ; x \in\right.$ $A\}$. For a distribution $T$ in $\Omega$ we set $\partial_{x_{i}} T(i=1, \ldots, d)$ for the weak first order derivatives, with similar notations for higher order derivatives.

Given $z_{0} \in L^{2}(\Omega), a \in L^{\infty, \infty}(Q), v \in L^{2}\left([0, T] ; H^{-1}(\Omega)\right)$ it is well known (see e.g. [34]) that the linear parabolic equation

$$
\begin{aligned}
\partial_{t} z-\Delta z+a(t, x) z & =v, \quad \text { in } Q \\
z & =0, \quad \text { in } \Sigma, \\
z(0) & =z_{0} \quad \text { in } \Omega,
\end{aligned}
$$

admits a unique solution $z \in W(0, T):=L^{2}\left([0, T] ; H_{0}^{1}(\Omega)\right) \cap W^{1,2}\left([0, T] ; H^{-1}(\Omega)\right)$ in the following weak sense: for a.a. $t \in[0, T]$ and $q \in H_{0}^{1}(\Omega)$ we have that

$$
\begin{aligned}
\left\langle\partial_{t} z(t), q\right\rangle_{H^{-1}(\Omega), H_{0}^{1}(\Omega)}+\langle\nabla z(t), \nabla q\rangle_{L^{2}(\Omega), L^{2}(\Omega)}+\langle a(t) z(t), q\rangle_{L^{2}(\Omega), L^{2}(\Omega)} & =\langle v(t), q\rangle_{H^{-1}(\Omega), L^{2}(\Omega)} \\
z(0) & =z_{0} .
\end{aligned}
$$


Note that the second line in $(2.2)$ is meaningful in view of the embedding $W(0, T) \hookrightarrow C\left([0, T] ; L^{2}(\Omega)\right)$ (see e.g. [34]). Moreover, there exists a constant $c>0$ such that

$$
\|z\|_{L^{2}\left([0, T] ; H^{1}(\Omega)\right)}+\left\|\partial_{t} z\right\|_{L^{2}\left([0, T] ; H^{-1}(\Omega)\right)} \leq c\left(\left\|z_{0}\right\|_{L^{2}(\Omega)}+\|v\|_{L^{2}\left([0, T] ; H^{-1}(\Omega)\right)}\right),
$$

and, in view of the integration by parts formula, for all $q \in W(0, T)$ we have that

$$
\begin{aligned}
& \int_{0}^{T}\left[-\left\langle\partial_{t} q(t), z(t)\right\rangle_{H^{-1}(\Omega), H_{0}^{1}(\Omega)}+\langle\nabla z(t), \nabla q(t)\rangle_{L^{2}(\Omega), L^{2}(\Omega)}+\langle a(t) z(t), q(t)\rangle_{L^{2}(\Omega), L^{2}(\Omega)}\right] \mathrm{d} t \\
& =\langle z(0), q(0)\rangle_{L^{2}(\Omega), L^{2}(\Omega)}-\langle z(T), q(T)\rangle_{L^{2}(\Omega), L^{2}(\Omega)}+\int_{0}^{T}\langle v(t), q(t)\rangle_{H^{-1}(\Omega), H_{0}^{1}(\Omega)} \mathrm{d} t .
\end{aligned}
$$

Now, for $s \in] 1, \infty[$ consider the space

$$
\mathcal{V}^{s}:=L^{s}\left([0, T] ; W^{2, s}(\Omega) \cap W_{0}^{1, s}(\Omega)\right) \cap W^{1, s}\left([0, T] ; L^{s}(\Omega)\right) .
$$

Endowed with the natural norm

$$
\|z\|_{\mathcal{V}^{s}}:=\|z\|_{s, s}+\left\|\partial_{t} z\right\|_{s, s}+\sum_{i=1}^{d}\left\|\partial_{x_{i}} z\right\|_{s, s}+\sum_{i, j=1}^{d}\left\|\partial_{x_{i} x_{j}} z\right\|_{s, s}
$$

$\mathcal{V}^{s}$ is a Banach space. It is well known that if the data $z_{0}, v$ are more regular than $L^{2}(\Omega)$ and $L^{2}\left([0, T] ; H^{-1}(\Omega)\right)$, respectively, then more regularity can be obtained for the solution $z$. We collect in the following proposition some useful results of this type. For simplicity we will assume that $z_{0} \equiv 0$, but similar results can be established for the more general case $z_{0} \in W_{0}^{2-\frac{2}{s}, s}(\Omega)$ (see [32, Chapter 4]).

Proposition 2.1. Let $s \in] 1, \infty\left[\right.$. Assume that $z_{0} \equiv 0$ and $v \in L^{s, s}(Q)$. Then, problem (2.1) admits a unique strong solution $z[v] \in \mathcal{V}^{s}$ (i.e. the equation is satisfied almost everywhere and $\left.z[v](0)=0\right)$. Moreover, the following assertion hold true:

(i) There exists a constant $c_{s}>0$ such that

$$
\|z[v]\|_{\mathcal{V}^{s}} \leq c_{s}\|v\|_{s, s}
$$

(ii) There exists a constant $c_{1}>0$ such that

$$
\|z[v](\cdot, T)\|_{1}+\|z[v]\|_{1,1} \leq c_{1}\|v\|_{1,1} .
$$

(iii) The linear application $v \in L^{2,2}(Q) \rightarrow z[v] \in L^{2,2}(Q) \cap C\left([0, T] ; L^{2}(\Omega)\right)$ is continuous when $L^{2,2}(Q)$ and $L^{2,2}(Q) \cap C\left([0, T] ; L^{2}(\Omega)\right)$ are respectively endowed with the weak and the strong (e.g. with the norm $\|\cdot\|_{2,2}+\|\cdot\|_{C\left([0, T] ; L^{2}(\Omega)\right)}$ for the latter $)$ topologies.

Proof. The existence and uniqueness of a strong solution and the estimates in assertion (i) are proved e.g. in [32, Chapter 4, Theorem 9.1]. Now, writing $z=z[v]$ for notational convenience, consider the equation

$$
\begin{aligned}
-\partial_{t} q-\Delta q+\alpha q & =\operatorname{sgn}(z) & & \text { in } Q \\
q & =0 & & \text { in } \Sigma, \\
q(T, \cdot) & =\operatorname{sgn}(z(T, \cdot)) & & \text { in } \Omega .
\end{aligned}
$$

This equation has a unique weak solution $q \in W(0, T)$. Moreover, since the terminal condition (which can be seen as an initial condition after the change of variable $t^{\prime}=T-t$ ) and the right hand side are bounded uniformly by 1 , by the maximum principle for weak solutions (see [32, Chapter 3 , 
Theorem 7.1]) we get that $\|q\|_{\infty, \infty}$ is bounded by a constant $c$ independent of $z$. Therefore, by (2.4), we obtain

$$
\int_{Q}|z(t, x)| \mathrm{d} t \mathrm{~d} x+\int_{\Omega}|z(T, x)| \mathrm{d} x=\int_{Q} v(t, x) q(t, x) \mathrm{d} t \mathrm{~d} x
$$

which together with $\|q\|_{\infty, \infty} \leq c$ imply (ii). Finally, if $v^{n} \rightarrow v$ weakly in $L^{2,2}(Q)$ by (i) we have that $z^{n}:=z\left[v^{n}\right]$ is bounded in $\mathcal{V}^{2}$. Therefore, there exists $\hat{z} \in \mathcal{V}^{2}$ such that, except for some subsequence, $z^{n} \rightarrow \hat{z}$ weakly in $L^{2}\left([0, T] ; H^{2}(\Omega)\right)$ and $\partial_{t} z^{n} \rightarrow \partial_{t} \hat{z}$ weakly in $L^{2,2}(Q)$. Therefore, passing to the limit in the first equation of (2.2) we get that $\hat{z}$ satisfies it and so $\hat{z}=z[v]$ by the uniqueness of weak solutions. On the other hand, since $H^{2}(\Omega)$ is compactly embedded in $L^{2}(\Omega)$, by Aubin's Theorem (see [2] and [33, Chapter 1, Théorème 5.1]), we have that $z^{n} \rightarrow z[v]$ strongly in $L^{2,2}(Q)$ and in $C\left([0, T] ; L^{2}(\Omega)\right)$. The result follows.

Remark 2.2. Note that by the embeddings $\mathcal{V}^{s} \hookrightarrow L^{s, s}(Q)$ and $\mathcal{V}^{s} \hookrightarrow C\left([0, T] ; L^{s}(\Omega)\right)($ for $s \in] 1, \infty[)$, and the proof of Proposition 2.1(ii) (for $s=1$ ) imply that

$$
\|z\|_{s, s} \leq c_{s}\|v\|_{s, s}, \quad \max _{t \in[0, T]}\|z(t, \cdot)\|_{s} \leq c_{s}\|v\|_{s, s}, \quad \forall s \in[1, \infty[.
$$

Given $u \in L^{\infty, \infty}(Q)$ and $\varphi: Q \times \mathbb{R} \times \mathbb{R} \rightarrow \mathbb{R}$, we consider the Cauchy problem

$$
\begin{aligned}
\partial_{t} y-\Delta y+\varphi(t, x, y, u) & =0 \quad \text { in } Q, \\
y & =0 \quad \text { in } \Sigma, \quad y(0, \cdot)=y_{0}(\cdot) \quad \text { in } \Omega .
\end{aligned}
$$

(H1) We assume that

(i) The initial condition $y_{0}$ belongs to $W_{0}^{2-\frac{2}{s}, s}(\Omega)$, with $s>(d+2) / 2$.

(ii) The function $\varphi$ is measurable and the following monotonicity-type property holds true: for all $R>0$ there exists $c=c(R)>0$ such that

$$
-c\left(1+|y|^{2}\right) \leq \varphi(t, x, y, u) y \text { for a.a. }(t, x, y) \in Q \times \mathbb{R},|u| \leq R .
$$

(iii) For a.a. $(t, x) \in Q$ the function $\varphi(t, x, \cdot, \cdot)$ is $C^{1}$ and for all $R>0$ there exists $c=c(R)$ such that for a.a $(t, x) \in Q$

$$
|\varphi(t, x, y, u)|+\left|\varphi_{y}(t, x, y, u)\right|+\left|\varphi_{u}(t, x, y, u)\right| \leq c \quad \text { if } \quad|(y, u)| \leq R .
$$

We recall that $y \in W(0, T)$ is a weak solution of (2.10) if for a.a. $t \in] 0, T$ [ and $q \in H_{0}^{1}(\Omega)$ we have that

$$
\begin{aligned}
\left\langle\partial_{t} y(t), q\right\rangle_{H^{-1}(\Omega), H_{0}^{1}(\Omega)}+\langle\nabla y(t), \nabla q\rangle_{L^{2}(\Omega), L^{2}(\Omega)}+\langle\varphi(t, \cdot, y(t), u(t)), q\rangle_{L^{2}(\Omega), L^{2}(\Omega)} & =0, \\
& y(0)=y_{0} .
\end{aligned}
$$

We say that $y$ is a strong solution if there exists $s \in] 1, \infty\left[\right.$ such that $y \in \mathcal{V}^{s}(Q)$. The following well-posedness result for (2.10) holds true:

Proposition 2.3. Under assumption (H1) for any $u \in L^{\infty, \infty}(Q)$, equation (2.10) admits a unique strong solution $y[u]$. Moreover, there exists $\beta>0$ such that $y[u] \in V^{s}(Q) \cap C^{\beta / 2, \beta}(\bar{Q})$ for all $\left.s \in\right] 1, \infty[$.

Proof. The existence and uniqueness of a weak solution $y[u] \in W(0, T)$ is based on standard arguments using the Garlekin's approximation, for the existence, and Gronwall Lemma, for the uniqueness (see [28, Proposition 2.1], in our framework, and [14, Theorem 5.1] for the case of homogeneous Neumann boundary conditions). On the other hand, by (H1)(i) and the Sobolev embeddings (see e.g. $[1,24,25])$ we have the existence of $\alpha>0$ such that $y_{0} \in C^{\alpha}(\bar{\Omega})$, which, by the results in [4], implies the existence of $\beta>0$ such that $y[u] \in C^{\beta / 2, \beta}(\bar{Q})$. Therefore, by (H1)(iii) we have that $\phi(\cdot, \cdot, y[u](\cdot, \cdot), u(\cdot, \cdot)) \in L^{\infty, \infty}(Q)$ and so the $\mathcal{V}^{s}(Q)$ regularity is a direct consequence of (H1)(i) and Proposition 2.1(i) 
Now, for $n_{E}, n_{I} \in \mathbb{N}$ let $\ell: Q \times \mathbb{R} \times \mathbb{R} \rightarrow \mathbb{R}, \Phi: \Omega \times \mathbb{R} \rightarrow \mathbb{R}, \Phi_{E}: \Omega \times \mathbb{R} \rightarrow \mathbb{R}^{n_{E}}$ and $\Phi_{I}: \Omega \times \mathbb{R} \rightarrow \mathbb{R}^{n_{I}}$ be such that:

(H2) The function $\ell$ is measurable and satisfies the same assumption stated for $\varphi$ in (H1)(iii). We suppose that $\psi=\Phi, \Phi_{E}, \Phi_{I}$ is measurable and for a.a. $x \in \Omega$ we have that $\psi(x, \cdot)$ is $C^{1}$ and there exists a constant $c=c(R)$ such that if $|y| \leq R$ then $|\psi(x, y)| \leq c$ and $\left|\psi_{y}(x, y)\right| \leq c$.

In order to perform a second order analysis we will also need the following assumption

(H3) For a.a. $(t, x) \in Q$ the functions $\varphi(t, x, \cdot, \cdot)$ and $\ell(t, x, \cdot, \cdot)$ are $C^{2}$. Moreover, there exists $c=c(R)$ such that for all $(y, u),\left(y^{\prime}, u^{\prime}\right)$, satisfying that $|(y, u)| \leq R$ and $\left|\left(y^{\prime}, u^{\prime}\right)\right| \leq R$, we have that

$$
\begin{gathered}
\left|\varphi_{(y, u)^{2}}(t, x, 0,0)\right|+\left|\ell_{(y, u)^{2}}(t, x, 0,0)\right| \leq c, \\
\left|\varphi_{(y, u)^{2}}(t, x, y, u)-\varphi_{(y, u)^{2}}\left(t, x, y^{\prime}, u^{\prime}\right)\right| \leq c\left(\left|y-y^{\prime}\right|+\left|u-u^{\prime}\right|\right), \\
\left|\ell_{(y, u)^{2}}(t, x, y, u)-\ell_{(y, u)^{2}}\left(t, x, y^{\prime}, u^{\prime}\right)\right| \leq c\left(\left|y-y^{\prime}\right|+\left|u-u^{\prime}\right|\right) .
\end{gathered}
$$

Analogously, for $\psi=\Phi, \Phi_{E}^{i}, \Phi_{I}^{j}\left(i=1, \ldots, n_{E}, j=1, \ldots, n_{I}\right)$, we suppose that for all $y, y^{\prime}$ with $|y| \leq R$ and $\left|y^{\prime}\right| \leq R$

$$
\left|\psi_{y y}(t, x, 0)\right| \leq c, \quad\left|\psi_{y y}(t, x, y)-\psi_{y y}\left(t, x, y^{\prime}\right)\right| \leq c\left|y-y^{\prime}\right| .
$$

Let us define $G_{E}: L^{\infty, \infty}(Q) \rightarrow \mathbb{R}^{n_{E}}, G_{I}: L^{\infty, \infty}(Q) \rightarrow \mathbb{R}^{n_{I}}$ as

$G_{E}^{i}(u):=\int_{\Omega} \Phi_{E}^{i}(x, y[u](T, x)) \mathrm{d} x \forall i=1, \ldots, n_{E}, G_{I}^{j}(u):=\int_{\Omega} \Phi_{I}^{j}(x, y[u](T, x)) \mathrm{d} x \forall j=1, \ldots, n_{I}$, and let $J: L^{\infty, \infty}(Q) \rightarrow \mathbb{R}$ and $G: L^{\infty, \infty}(Q) \rightarrow \mathbb{R}^{n_{E}} \times \mathbb{R}^{n_{I}}$ be defined as

$$
J(u):=\int_{Q} \ell(t, x, y[u](t, x), u(t, x)) \mathrm{d} x \mathrm{~d} t+\int_{\Omega} \Phi(x, y[u](T, x)) \mathrm{d} x, \quad G(u):=\left(G_{E}(u), G_{I}(u)\right) .
$$

Note that under (H2) the above functions are well defined. Now, given $a, b \in L^{\infty, \infty}(Q)$ satisfying that $a \leq b$ a.e. in $Q$ and $\operatorname{essinf}(b(t, x)-a(t, x))>0$, let us define the sets

$$
\begin{aligned}
& \mathcal{K}_{1}=\left\{u \in L^{\infty, \infty}(Q) \mid a(t, x) \leq u(t, x) \leq b(t, x), \text { for a.a. }(t, x) \in Q\right\}, \\
& \mathcal{K}_{2}=\{0\}^{n_{E}} \times \mathbb{R}_{-}^{n_{I}}, \quad \mathcal{K}=\mathcal{K}_{1} \cap G^{-1}\left(\mathcal{K}_{2}\right) .
\end{aligned}
$$

We consider the problem

$$
\inf _{u \in L^{\infty, \infty}(Q)} J(u) \text { subject to } u \in \mathcal{K} .
$$

Definition 2.4. (i) We say that $\bar{u} \in \mathcal{K}$ is a weak local solution of $(P)$ if there exists $\varepsilon>0$ such that $J(u) \geq J(\bar{u})$ for all $u \in \mathcal{K}$ such that $\|u-\bar{u}\|_{\infty, \infty} \leq \varepsilon$.

(ii) We say that $J$ satisfies a local quadratic growth condition in the weak sense at $\bar{u} \in \mathcal{K}$ if there exists $\alpha, \varepsilon>0$ such that

$$
J(u) \geq J(\bar{u})+\frac{\alpha}{2}\|u-\bar{u}\|_{2,2}^{2} \quad \text { for all } u \in \mathcal{K} \text { such that }\|u-\bar{u}\|_{\infty, \infty} \leq \varepsilon .
$$

(iii) We say that $J$ satisfies a local quadratic growth condition in the $L^{s}$-weak sense $(s \in[1, \infty[)$ at $\bar{u} \in \mathcal{K}$ if there exists $\alpha, \varepsilon>0$ such that

$$
J(u) \geq J(\bar{u})+\frac{\alpha}{2}\|u-\bar{u}\|_{2,2}^{2} \quad \text { for all } u \in \mathcal{K} \text { such that }\|u-\bar{u}\|_{s, s} \leq \varepsilon .
$$

Remark 2.5. Since $\mathcal{K}$ is bounded in $L^{\infty, \infty}(Q)$, for any $s \in\left[1, \infty\left[\right.\right.$ the topology of $L^{s, s}(Q)$ restricted to $\mathcal{K}$ is equivalent to the topology of $L^{1,1}(Q)$ restricted to $\mathcal{K}$ (see [3, Remark 2.7]). This implies that $\bar{u}$ satisfies the $L^{s, s}$-weak quadratic growth condition (2.14) iff $\bar{u}$ satisfies the $L^{1,1}$-weak quadratic growth condition. 


\section{Second order expansions for the Lagrangian associated to $(P)$}

We first prove some Taylor type expansion for the function $u \in L^{\infty, \infty}(Q) \rightarrow y[u] \in \mathcal{V}^{s} \cap C(\bar{Q})$ $(s \in] 1, \infty[)$. Let us fix $\bar{u} \in L^{\infty, \infty}(Q)$ and set $\bar{y}:=y[\bar{u}], \bar{\varphi}(t, x):=\varphi(t, x, \bar{y}(t, x), \bar{u}(t, x))$. For notational convenience we also write

$$
\begin{gathered}
\bar{\varphi}_{y}(t, x):=\varphi_{y}(t, x, \bar{y}(t, x), \bar{u}(t, x)), \quad \bar{\varphi}_{u}(t, x):=\varphi_{u}(t, x, \bar{y}(t, x), \bar{u}(t, x)), \\
\bar{\varphi}_{(y, u)}(t, x)(z, v)=\bar{\varphi}_{y}(t, x) z+\bar{\varphi}_{u}(t, x) v \quad \forall(z, v) \in \mathbb{R}^{2},
\end{gathered}
$$

with a similar notation for the second order derivatives evaluated at $(t, x, \bar{y}(t, x), \bar{u}(t, x))$. Given $v \in L^{\infty, \infty}(Q)$ define $z_{1}[v] \in \mathcal{V}^{s}(Q)$ as the unique solution of

$$
\begin{aligned}
\partial_{t} z-\Delta z+\bar{\varphi}_{(y, u)}(t, x)(z, v) & =0 \text { in } Q, \\
z & =0 \text { in } \Sigma, \\
z(0, \cdot) & =0 \text { in } \Omega .
\end{aligned}
$$

Note that under $(\mathbf{H 1})(\mathrm{iii}), z_{1}[v]$ is well-defined. We now prove some technical results:

Lemma 3.1. Let us set $\delta y=y[\bar{u}+v]-y[\bar{u}]$. Then,

$$
\begin{aligned}
\left\|z_{1}[v]\right\|_{\mathcal{V}^{s}}+\|\delta y\|_{\mathcal{V}^{s}} & =O\left(\|v\|_{s, s}\right), \text { for all } s \in[1, \infty[ \\
\left\|\delta y-z_{1}[v]\right\|_{s, s} & =O\left(\left\|(\delta y)^{2}\right\|_{s, s}+\left\|v^{2}\right\|_{s, s}\right), \text { for all } s \in[1, \infty[, \\
\left\|\delta y(T, \cdot)-z_{1}[v](T, \cdot)\right\|_{s} & =O\left(\left\|(\delta y)^{2}\right\|_{s, s}+\left\|v^{2}\right\|_{s, s}\right) \text { for all } s \in[1, \infty[.
\end{aligned}
$$

Proof. The estimate for $\left\|z_{1}[v]\right\|_{\mathcal{V}}$ is an immediate consequence of (3.2), Proposition 2.1(i) and (2.9). Let us set $\delta \varphi(t, x):=\varphi(t, x, y[\bar{u}+v](t, x), \bar{u}(t, x)+v(t, x))-\bar{\varphi}(t, x)$. By definition and Proposition 2.3 we have that $\delta y$ satisfies the following equation in the strong sense

$$
\begin{aligned}
\partial_{t} \delta y-\Delta \delta y+\delta \varphi(t, x) & =0 \text { in } Q, \\
\delta y & =0 \text { in } \Sigma, \\
\delta y(0, \cdot) & =0 \text { in } \Omega .
\end{aligned}
$$

Expanding $\delta \varphi(t, x)$, the first equation in (3.4) becomes

$$
\partial_{t} \delta y-\Delta \delta y+\int_{0}^{1} \varphi_{(y, u)}(t, x, \bar{y}+\tau \delta y, \bar{u}+\tau v)(\delta y, v) \mathrm{d} \tau=0, \text { for a.a. }(t, x) \in Q .
$$

Therefore, by Proposition 2.1(i) and (H1)(iii), we obtain the estimates for $\|\delta y\|_{\mathcal{V}^{s}}$ in the first equation of (3.3). Now, set $d_{1}:=y[\bar{u}+v]-y[\bar{u}]-z_{1}[v]$. Then, $d_{1}$ satisfies the equation

$$
\begin{aligned}
\partial_{t} d_{1}-\Delta d_{1}+\bar{\varphi}_{y}(t, x) d_{1}+\delta \varphi(t, x)-\bar{\varphi}_{(y, u)}(t, x)(\delta y, v) & =0 \text { in } Q, \\
d_{1} & =0 \text { in } \Sigma \\
d_{1}(0, \cdot) & =0 \text { in } \Omega .
\end{aligned}
$$

Expanding $\delta \varphi(t, x)$, as before, the first equation in (3.5) becomes

$$
\partial_{t} d_{1}-\Delta d_{1}+\bar{\varphi}_{y}(t, x) d_{1}+\int_{0}^{1}\left[\varphi_{(y, u)}(t, x, \bar{y}+\tau \delta y, \bar{u}+\tau v)-\bar{\varphi}_{(y, u)}(t, x)\right](\delta y, v) \mathrm{d} \tau=0 .
$$

and the second and third equation (3.5) follow from (H1)(iii), Proposition 2.1(i) and (2.9). 
Now, we study second order expansions. Let us set $z_{2}[v] \in \mathcal{V}^{s}$ for the unique solution of

$$
\begin{aligned}
\partial_{t} z-\Delta z+\bar{\varphi}_{y}(t, x) z+\frac{1}{2} \bar{\varphi}_{(y, u)^{2}}(t, x)\left(z_{1}[v], v\right)^{2} & =0 \text { in } Q, \\
z & =0 \text { in } \Sigma, \\
z(0, \cdot) & =0 \text { in } \Omega .
\end{aligned}
$$

Note that under $(\mathbf{H} 3)$, the estimates for $z_{1}[v]$ in Lemma 3.1 imply that $z_{2}[v]$ is well defined.

Lemma 3.2. For all $s \in] 1, \infty[$, we have the following estimates:

$$
\begin{aligned}
\left\|z_{2}[v]\right\|_{s, s} & =O\left(\left\|\left|z_{1}(v)\right|^{2}\right\|_{s, s}+\left\||v|^{2}\right\|_{s, s}\right), \\
\left.\| y[\bar{u}+v]-y[\bar{u}]-z_{1}[v]-z_{2}[v]\right] \|_{1,1} & =O\left(\|v\|_{\infty, \infty}\|v\|_{2,2}^{2}\right), \\
\left.\| y[\bar{u}+v](T, \cdot)-y[\bar{u}](T, \cdot)-z_{1}[v](T, \cdot)-z_{2}[v]\right](T, \cdot) \|_{1} & =O\left(\|v\|_{\infty, \infty}\|v\|_{2,2}^{2}\right) .
\end{aligned}
$$

Proof. As before, we set $\delta y=y[\bar{u}+v]-y[\bar{u}]$. The first estimate follows directly from Proposition 2.1(i) and (H3). For notational convenience we set $d_{2}:=\delta y-z_{1}[v]-z_{2}[v]=d_{1}-z_{2}[v]$. By (3.5) and (3.7), $d_{2}$ satisfies

$$
\partial_{t} d_{2}-\Delta d_{2}+\bar{\varphi}_{y}(t, x) d_{2}+\delta \varphi(t, x)-\bar{\varphi}_{(y, u)}(t, x)(\delta y, v)-\frac{1}{2} \bar{\varphi}_{(y, u)^{2}}(t, x)\left(z_{1}[v], v\right)^{2}=0 \text { in } Q,
$$

with zero boundary conditions. By a Taylor expansion we easily check, omitting the $(t, x)$ argument,

$$
\begin{aligned}
\delta \varphi-\bar{\varphi}_{(y, u)}(\delta y, v)-\frac{1}{2} \bar{\varphi}_{(y, u)^{2}}\left(z_{1}[v], v\right)^{2}= & \int_{0}^{1}(1-\tau)\left[\varphi_{(y, u)^{2}}(t, x, \bar{y}+\tau \delta y, \bar{u}+\tau v)-\bar{\varphi}_{(y, u)^{2}}\right](\delta y, v)^{2} \mathrm{~d} \tau \\
& +O\left(\left|d_{1}\right||\delta y|+\left|d_{1}\right|\left|z_{1}[v]\right|+\left|d_{1}\right||v|\right),
\end{aligned}
$$

the result follows from (H3), Proposition 2.1(ii), the estimates for $\delta y$ and the $L^{1}$-estimates for $d_{1}$ in Lemma 3.1 (take $s=1$ in the second and third equations of (3.1) and use that $\|\delta y\|_{\infty, \infty} \leq c\|v\|_{\infty, \infty}$, $\left\|z_{1}[v]\right\|_{\infty, \infty} \leq c\|v\|_{\infty, \infty}$, for some $c>0$ by the maximum principle).

Let us define the Lagrangian $\mathcal{L}: L^{\infty, \infty}(Q) \times \mathbb{R}^{n_{E}} \times \mathbb{R}^{n_{I}} \rightarrow \mathbb{R}$ associated to $(P)$ as

$$
\mathcal{L}(u, \lambda):=J(u)+\lambda^{\top} G(u) \quad \text { where } \lambda=\left(\lambda_{E}, \lambda_{I}\right) \in \mathbb{R}^{n_{E}} \times \mathbb{R}^{n_{I}} .
$$

Using the estimates in Lemma 3.1 and Lemma 3.2 we study now a second order expansion for $\mathcal{L}(\cdot, \lambda)$. As for $\varphi$, for notational convenience we set $\bar{\ell}(t, x):=\ell(t, x, \bar{y}(t, x), \bar{u}(t, x))$ and

$$
\bar{\ell}_{y}(t, x):=\ell_{y}(t, x, \bar{y}(t, x), \bar{u}(t, x)), \quad \bar{\ell}_{u}(t, x):=\ell_{u}(t, x, \bar{y}(t, x), \bar{u}(t, x)),
$$

with a similar notation for the second order derivatives. Given $\lambda=\left(\lambda_{E}, \lambda_{I}\right) \in \mathbb{R}^{n_{E}} \times \mathbb{R}^{n_{I}}$ we set

$$
\Phi[\lambda](x, y):=\Phi(x, y)+\lambda_{E}^{\top} \Phi_{E}(x, y)+\lambda_{I}^{\top} \Phi_{I}(x, y) \text { for all } y \in \mathbb{R}, x \in \Omega
$$

As before, we denote $\bar{\Phi}[\lambda](x):=\Phi[\lambda](x, \bar{y}(T, x))$, with analogous notations for the derivatives. We have the following second order expansion of the Lagrangian.

Proposition 3.3. Let $C \subseteq \mathbb{R}^{n_{E}} \times \mathbb{R}^{n_{I}}$ be a nonempty compact set. Then, for every $\lambda=\left(\lambda_{E}, \lambda_{I}\right) \in C$ and $v \in L^{\infty, \infty}(Q)$ we have that

$$
\begin{aligned}
\mathcal{L}(\bar{u}+v, \lambda)= & \mathcal{L}(\bar{u}, \lambda)+\int_{Q}\left[\bar{\ell}_{y}(t, x)\left[z_{1}[v]+z_{2}[v]\right]+\bar{\ell}_{u}(t, x) v+\frac{1}{2} \bar{\ell}_{(y, u)^{2}}(t, x)\left(z_{1}[v], v\right)^{2}\right] \mathrm{d} t \mathrm{~d} x \\
& \left.+\int_{\Omega}\left\{\bar{\Phi}_{y}[\lambda](x)\left[z_{1}[v](T, x)+z_{2}[v](T, x)\right]+\frac{1}{2} \bar{\Phi}_{y y}[\lambda](x)\left(z_{1}[v](T, x)\right]\right)^{2}\right\} \mathrm{d} x \\
& +O\left(\|v\|_{\infty, \infty}\|v\|_{2,2}^{2}\right)
\end{aligned}
$$

where $O\left(\|v\|_{\infty, \infty}\|v\|_{2,2}^{2}\right)$ is uniform for $\lambda \in C$. 
Proof. Denoting $\delta y=y[\bar{u}+v]-\bar{y}$, by $(\mathbf{H 2})-(\mathbf{H 3})$ and the fact that $\lambda \in C$, we have that

$$
\begin{aligned}
\mathcal{L}(u+v, \lambda)= & \mathcal{L}(\bar{u}, \lambda)+\int_{Q}\left[\bar{\ell}_{y}(t, x) \delta y+\bar{\ell}_{u}(t, x) v+\frac{1}{2} \bar{\ell}_{(y, u)^{2}}(t, x)(\delta y, v)^{2}\right] \mathrm{d} t \mathrm{~d} x \\
& +\int_{\Omega}\left[\bar{\Phi}_{y}[\lambda](x) \delta y(T, x)+\frac{1}{2} \bar{\Phi}_{y y}[\lambda](x)(\delta y(T, x))^{2}\right] \mathrm{d} x \\
& +O\left(\int_{Q}\left(|\delta y|^{3}+|\delta y|^{2}|v|+|\delta y||v|^{2}+|v|^{3}\right) \mathrm{d} t \mathrm{~d} x+\int_{\Omega}|\delta y(T, x)|^{3} \mathrm{~d} x\right)
\end{aligned}
$$

where the $O(\cdot)$ term is uniform in $\lambda \in C$. Using the estimates in Lemma 3.1 and Proposition 2.1(i), we have that the expression inside the $O$ term is a $O\left(\|v\|_{\infty, \infty}\|v\|_{2,2}^{2}\right)$. Denoting by $d_{1}:=\delta y-z_{1}[v]$ and $d_{2}:=\delta y-z_{1}[v]-z_{2}[v]$, we get from (3.12)

$$
\begin{aligned}
\mathcal{L}(\bar{u}+v, \lambda)= & \mathcal{L}(\bar{u}, \lambda)+\int_{Q}\left[\bar{\ell}_{y}(t, x)\left[z_{1}[v]+z_{2}[v]\right]+\bar{\ell}_{u}(t, x) v+\frac{1}{2} \bar{\ell}_{(y, u)^{2}}(t, x)\left(z_{1}[v], v\right)^{2}\right] \mathrm{d} t \mathrm{~d} x \\
& \left.+\int_{\Omega}\left\{\bar{\Phi}_{y}[\lambda](x)\left[z_{1}[v](T, x)+z_{2}[v](T, x)\right]+\frac{1}{2} \bar{\Phi}_{y y}[\lambda](x)\left(z_{1}[v](T, x)\right]\right)^{2}\right\} \mathrm{d} x \\
& +O\left(\|v\|_{\infty, \infty}\|v\|_{2,2}^{2}\right)+O\left(\int_{Q}\left[\left|d_{2}\right|+\left|d_{1}\right|^{2}+\left|z_{1}[v]\right|\left|d_{1}\right|+\left|d_{1}\right||v|\right] \mathrm{d} t \mathrm{~d} x\right) \\
& +O\left(\int_{\Omega}\left[\left|d_{2}(x, T)\right|+\left|d_{1}(x, T)\right|^{2}+\left|d_{1}(x, T) \| z_{1}(x, T)\right|\right] \mathrm{d} x\right) .
\end{aligned}
$$

By Lemma 3.1 and Lemma 3.2 we obtain

$\int_{Q}\left[\left|d_{2}\right|+\left|d_{1}\right|^{2}+\left|z_{1}[v]\right|\left|d_{1}\right|+\left|d_{1}\right||v|\right] \mathrm{d} t \mathrm{~d} x+\int_{\Omega}\left[\left|d_{2}(x, T)\right|+\left|d_{1}(x, T)\right|^{2}+\left|d_{1}(x, T) \| z_{1}(x, T)\right|\right] \mathrm{d} x=O\left(\|v\|_{\infty, \infty}\|v\|_{2,2}^{2}\right)$,

which implies the result.

Remark 3.4. In particular, since the $O\left(\|v\|_{\infty, \infty}\|v\|_{2,2}^{2}\right)$ is also a $O\left(\|v\|_{\infty, \infty}^{3}\right)$, it is easy to see that restricted to $L^{\infty, \infty}(Q)$ the function $\mathcal{L}(\cdot, \lambda)$ is $C^{2}$ with

$$
\begin{aligned}
D_{u} \mathcal{L}(\bar{u}, \lambda) v= & \int_{Q}\left[\bar{\ell}_{y}(t, x) z_{1}[v]+\bar{\ell}_{u}(t, x) v\right] \mathrm{d} t \mathrm{~d} x+\int_{\Omega} \Phi_{y}[\lambda](x, \bar{y}(T, x)) z_{1}[v](T, x) \mathrm{d} x, \\
\frac{1}{2} D_{u u}^{2} \mathcal{L}(\bar{u}, \lambda) v^{2}= & \int_{Q}\left[\bar{\ell}_{y}(t, x) z_{2}[v]+\frac{1}{2} \bar{\ell}_{(y, u)^{2}}(t, x)\left(z_{1}[v], v\right)^{2}\right] \mathrm{d} t \mathrm{~d} x \\
& \left.+\int_{\Omega}\left[\Phi_{y}[\lambda](x, \bar{y}(T, x)) z_{2}[v](T, x)+\frac{1}{2} \Phi_{y y}[\lambda](x, \bar{y}(T, x))\left(z_{1}[v](T, x)\right]\right)^{2}\right\} \mathrm{d} x .
\end{aligned}
$$

Moreover, by assumption (H2) and the estimates in Lemma 3.3 and Lemma 3.2, we have that

$$
D_{u} \mathcal{L}(\bar{u}, \lambda) v=O\left(\|v\|_{1,1}\right), \quad D_{u u}^{2} \mathcal{L}(\bar{u}, \lambda) v^{2}=O\left(\|v\|_{2,2}^{2}\right) .
$$

This implies that $D_{u} \mathcal{L}(\bar{u}, \lambda)$ and the quadratic form $D_{u u}^{2} \mathcal{L}(\bar{u}, \lambda) v^{2}$ admit unique extensions to $L^{1,1}(Q)$ and $L^{2,2}(Q)$, respectively. By taking $\lambda=0$, the same extensions apply for $D J(\bar{u}) v$ and $D^{2} J(\bar{u}) v^{2}$.

The Hamiltonian $H: Q \times \mathbb{R} \times \mathbb{R} \times \mathbb{R} \rightarrow \mathbb{R}$ associated to problem $(P)$ is defined as

$$
H(t, x, y, p, u):=\ell(t, x, y, u)-p \varphi(t, x, y, u) .
$$

Let us define the adjoint state $\bar{p}_{\lambda} \in W(0, T)$ associated to $\bar{u}$ as the unique solution of

$$
\begin{aligned}
-\partial_{t} p-\Delta p-H_{y}(t, x, \bar{y}, p, \bar{u}) & =0 \text { in } Q, \\
p & =0 \text { in } \Sigma, \\
p(T, \cdot) & =\bar{\Phi}_{y}[\lambda](\cdot) .
\end{aligned}
$$

Since $u \in \mathcal{K}_{1}$, by (H1)-(H2), we have that $\varphi(\cdot, \cdot, \bar{y}, \bar{u}), \ell_{y}(\cdot, \cdot, \bar{y}, \bar{u}) \in L^{\infty, \infty}(Q)$ and $\Phi_{y}[\lambda](\cdot, \bar{y}(T, \cdot)) \in$ $L^{\infty}(\Omega)$. Thus, by maximum principle we get that $\bar{p}_{\lambda} \in L^{\infty, \infty}(Q)$. Let us set

$$
\begin{gathered}
\bar{H}[\lambda](t, x):=H_{y}\left(t, x, \bar{y}(t, x), \bar{p}_{\lambda}(t, x), \bar{u}(t, x)\right), \\
\bar{H}_{y}[\lambda](t, x):=H_{y}\left(t, x, \bar{y}(t, x), \bar{p}_{\lambda}(t, x), \bar{u}(t, x)\right), \quad \bar{H}_{u}[\lambda](t, x):=H_{u}\left(t, x, \bar{y}(t, x), \bar{p}_{\lambda}(t, x), \bar{u}(t, x)\right),
\end{gathered}
$$


with similar notations for the second order derivatives. We define the linear form $Q_{1}: L^{1,1}(Q) \rightarrow \mathbb{R}$ and the quadratic form $Q_{2}: L^{2,2}(Q) \rightarrow \mathbb{R}$ as

$$
\begin{aligned}
Q_{1}[\bar{u}, \lambda] v & :=\int_{Q} \bar{H}_{u}[\lambda](t, x) v(t, x) \mathrm{d} t \mathrm{~d} x, \\
Q_{2}[\bar{u}, \lambda](v) & :=\int_{Q} \bar{H}_{(y, u)^{2}}[\lambda](t, x)\left(z_{1}[v], v\right)^{2} \mathrm{~d} t \mathrm{~d} x+\int_{\Omega} \bar{\Phi}_{y y}[\lambda](x)\left(z_{1}[v](T, x)\right)^{2} \mathrm{~d} x .
\end{aligned}
$$

Since $\bar{p}_{\lambda} \in L^{\infty, \infty}(Q)$, we have that $Q_{1}[\bar{u}, \lambda](v)$ and $Q_{2}[\bar{u}, \lambda](v)$ are well defined. The following result is a consequence of Proposition 3.3 and the integration by parts formula (2.4).

Proposition 3.5. Let $(\lambda, C, v)$ be as in Proposition 3.3. Then

$$
D_{u} \mathcal{L}(\bar{u}, \lambda) v=Q_{1}[\bar{u}, \lambda] v \text { and } D_{u u} \mathcal{L}(\bar{u}, \lambda) v^{2}=Q_{2}[\bar{u}, \lambda](v) .
$$

As a consequence,

$$
\mathcal{L}(\bar{u}+v, \lambda)=\mathcal{L}(\bar{u}, \lambda)+Q_{1}[\bar{u}, \lambda] v+\frac{1}{2} Q_{2}[\bar{u}, \lambda](v)+O\left(\|v\|_{\infty, \infty}\|v\|_{2,2}^{2}\right),
$$

where the $O\left(\|v\|_{\infty, \infty}\|v\|_{2,2}^{2}\right)$ term is uniform for $\lambda \in C$.

Proof. We have that

$$
\int_{\Omega} \bar{\Phi}_{y}[\lambda](x) z_{1}[v](T, x) \mathrm{d} x=\int_{\Omega} \bar{p}_{\lambda}(T, x) z_{1}[v](T, x) \mathrm{d} x .
$$

Letting $z=z_{1}[v]$ and $q=\bar{p}_{\lambda}$ in $(2.4)$, and setting $\langle\cdot, \cdot\rangle_{*}=\langle\cdot, \cdot\rangle_{H^{-1}(\Omega), H_{0}^{1}(\Omega)}$ and $\langle\cdot, \cdot\rangle_{0}:=\langle\cdot, \cdot\rangle_{L^{2}(\Omega), L^{2}(\Omega)}$, we obtain that

$$
\left\langle\bar{p}_{\lambda}(T, \cdot) z_{1}[v](T, \cdot)\right\rangle_{0}=\int_{0}^{T}\left[\left\langle\partial_{t} \bar{p}_{\lambda}, z_{1}[v]\right\rangle_{*}-\left\langle\nabla z_{1}[v], \nabla \bar{p}_{\lambda}\right\rangle_{0}-\left\langle\bar{\varphi}_{y}(t, \cdot) z_{1}[v], \bar{p}_{\lambda}\right\rangle_{0}-\left\langle\bar{\varphi}_{u}(t, \cdot) v, \bar{p}_{\lambda}\right\rangle_{0}\right] \mathrm{d} t .
$$

By (3.16), for a.a. $t \in] 0, T[$ we have that

$$
\left\langle\partial_{t} \bar{p}_{\lambda}(t, \cdot), z_{1}[v](t, \cdot)\right\rangle_{*}=\left\langle\nabla \bar{p}_{\lambda}(t, \cdot), \nabla z_{1}[v](t, \cdot)\right\rangle_{0}+\left\langle\bar{p}_{\lambda}(t, \cdot) \bar{\varphi}_{y}(t, \cdot)-\bar{\ell}_{y}(t, \cdot), z_{1}[v](t, \cdot)\right\rangle_{0},
$$

which implies that

$$
\left\langle\bar{p}_{\lambda}(T, \cdot) z_{1}[v](T, \cdot)\right\rangle_{0}=-\int_{0}^{T}\left[\left\langle\bar{\ell}_{y}(t, \cdot), z_{1}[v](t, \cdot)\right\rangle_{0}+\left\langle\bar{\varphi}_{u}(t, \cdot) v, \bar{p}_{\lambda}\right\rangle_{0}\right] \mathrm{d} t .
$$

Using the expression above, (3.19) and the first equation in (3.14), we obtain the first identity in (3.18). The second identity in (3.18) is proved by an analogous argument using the equation for $z_{2}[v]$ and the equation for $\bar{p}_{\lambda}$.

\section{First order optimality conditions}

In this section we recall some classical results on first optimality conditions for problem $(P)$. We recall that for any Banach space $\left(X,\|\cdot\|_{X}\right)$ and $K \subseteq X$ the radial and tangent cone to $K$ at point $u$ are defined respectively as

$$
\begin{aligned}
R_{K}(u) & :=\{v \in X ; \exists \hat{\tau}>0 \text { such that } u+\tau v \in K, \text { for } \tau \in[0, \hat{\tau}] \geq 0\}, \\
T_{K}(u) & :=\left\{v \in X \mid \exists u(\sigma)=u+\tau v+o(\tau) \in K, \tau>0,\|o(\tau) / \tau\|_{X} \rightarrow 0, \text { as } \sigma \downarrow 0\right\} .
\end{aligned}
$$

We recall also that if $K$ is convex, then $T_{K}(u)=\operatorname{clos}_{X}\left(R_{K}(u)\right)$, where $\operatorname{clos}_{X}(A)$ denotes the closure in $X$ of $A \subseteq X$. Let us fix $\bar{u} \in \mathcal{K}$ and set $\bar{y}=y[\bar{u}]$.

We will need the following assumption ([37]):

(H3) The following Robinson constraint qualification condition holds true

$$
0 \in \operatorname{int}\left\{G(\bar{u})+D G(\bar{u})\left(\mathcal{K}_{1}-\bar{u}\right)-\mathcal{K}_{2}\right\} .
$$


Remark 4.1. Note that $(R C Q)$ implies in particular that

$$
\mathbb{R}^{n_{E}} \times \mathbb{R}^{n_{I}}=D G(\bar{u}) R_{\mathcal{K}_{1}}(\bar{u})-R_{\mathcal{K}_{2}}(G(\bar{u})) .
$$

We define the following active sets

$$
\begin{gathered}
A_{a}(\bar{u}):=\{(t, x) \in Q ; \bar{u}(t, x)=a(t, x)\}, \quad A_{b}(\bar{u}):=\{(t, x) \in Q ; \bar{u}(t, x)=b(t, x)\}, \\
I^{1}(\bar{u}):=\left\{j \in\left\{1, \ldots, n_{I}\right\} ; G_{I}^{j}(\bar{u})=0\right\} .
\end{gathered}
$$

Note that $T_{[a(t, x), b(t, x)]}(\bar{u}(t, x))$ is equal to $\mathbb{R}_{+}$in $A_{a}(\bar{u})$, is equal to $\mathbb{R}_{-}$in $A_{b}(\bar{u})$ and is equal to $\mathbb{R}$ otherwise.

Lemma 4.2. Under (H3), we have that

$$
T_{\mathcal{K}}(\bar{u}):=\left\{v \in L^{2,2}(Q) ; v(t, x) \in T_{[a(t, x), b(t, x)]}(\bar{u}(t, x)), D G_{E}(\bar{u}) v=0, D G_{I}^{i}(\bar{u}) v \leq 0 \forall i \in I^{1}(\bar{u})\right\},
$$

when $\mathcal{K}$ is considered as a subset of $X=L^{2,2}(Q)$.

Proof. Let us call $\hat{T}_{\mathcal{K}}(\bar{u})$ the r.h.s. of (4.4). If $v \in T_{\mathcal{K}}(\bar{u})$ then there exists $r: \mathbb{R}_{+} \rightarrow L^{2,2}(Q)$, satisfying that, $\|r(\tau) / \tau\|_{2,2} \rightarrow 0$ as $\tau \downarrow 0$, such that $u(\tau):=\bar{u}+\tau v+r(\tau) \in \mathcal{K}$. Thus, a.e. in $Q$,

$$
\begin{gathered}
a(t, x) \leq \bar{u}(t, x)+\tau v(t, x)+r(\tau)(t, x) \leq b(t, x), G_{E}(u(\tau))=\tau D G_{E}(\bar{u}) v+o(\tau)=0, \\
G_{I}^{j}(u(\tau))=\tau D G_{I}^{j}(\bar{u}) v+o(\tau) \leq 0, \text { for all } j \in I(\bar{u}) .
\end{gathered}
$$

The expansions for $G_{E}(u(\tau))$ and $G_{I}^{i}(u(\tau))$ are justified by the results in the previous section and the fact that $\tau v+r(\tau) \in \mathcal{K}-\bar{u} \subseteq L^{\infty, \infty}(Q)$. In fact, setting $\hat{v}_{\tau}=\tau v+r(\tau)$ we have that $\left\|\hat{v}_{\tau}\right\|_{\infty, \infty}$ is uniformly bounded and so $O\left(\left\|\hat{v}_{\tau}\right\|_{\infty, \infty}\left\|\hat{v}_{\tau}\right\|_{2,2}^{2}\right)=o(\tau)$. The terms $D^{2} G_{E}(\bar{u}) \hat{v}_{\tau}^{2}$ and $D^{2} G_{I}(\bar{u}) \hat{v}_{\tau}^{2}$ are clearly a $O\left(\tau^{2}\right)$, which justifies the expansion. Now, considering first $(t, x) \in A_{a}(\bar{u})$ and then $(t, x) \in A_{b}(\bar{u})$, dividing by $\tau$, letting $\tau \downarrow 0$ in the above expressions and using that up to some subsequence, $r(\tau)(t, x) / \tau \rightarrow 0$ a.e. we get that $v \in \hat{T}_{\mathcal{K}}(\bar{u})$. Conversely, let $v \in \hat{T}_{\mathcal{K}}(\bar{u})$ and for $\sigma>0$ set

$$
v^{\sigma}:=\frac{P_{\mathcal{K}_{1}}(\bar{u}+\sigma v)-\bar{u}}{\sigma},
$$

where $P_{\mathcal{K}_{1}}$ is the projection operator in $L^{2,2}(Q)$ onto the closed and convex set $\mathcal{K}_{1}$. By the results in [38] we have that $v^{\sigma}$ is measurable. Moreover, a.e. in $Q$

$$
v^{\sigma}(t, x):=\frac{P_{[a(t, x), b(t, x)]}(\bar{u}(t, x)+\sigma v(t, x))-\bar{u}(t, x)}{\sigma},
$$

where for $a, b \in \mathbb{R}$, such that $a \leq b$, and $c \in \mathbb{R}, P_{[a, b]}(c)$ is equal to $c$ if $c \in[a, b]$, is equal to $a$ if $c<a$ and is equal to $b$ otherwise. By definition, $v^{\sigma} \in R_{\mathcal{K}_{1}}(\bar{u})$ and by Lebesgue Theorem, using that (4.5) implies that $\left|v^{\sigma}(t, x)\right| \leq|v(t, x)|$, we get that $\left\|v^{\sigma}-v\right\|_{2,2} \rightarrow 0$, as $\sigma \rightarrow 0$. In particular, setting $u^{\sigma}=\bar{u}+\sigma v^{\sigma}$, the expansions in the previous section and the fact that $\sigma v^{\sigma}$ is uniformly bounded in $L^{\infty, \infty}(Q)$ imply again that

$$
G_{E}\left(u^{\sigma}\right)=G_{E}(\bar{u})+\sigma D G_{E}(\bar{u}) v^{\sigma}+o(\sigma), G_{I}\left(u^{\sigma}\right)=G_{I}(\bar{u})+\sigma D G_{I}(\bar{u}) v^{\sigma}+o(\sigma) .
$$

Since $\left\|v^{\sigma}-v\right\|_{1,1} \rightarrow 0$ we have that $D G_{E}(\bar{u}) v^{\sigma}=D G_{E}(\bar{u}) v+o(1)$ and $D G_{I}(\bar{u}) v^{\sigma}=D G_{I}(\bar{u}) v+o(1)$. Therefore, $G_{E}\left(u^{\sigma}\right)=o(\sigma)$ and $G_{I}\left(u^{\sigma}\right) \leq o(\sigma)$, which implies that $d_{L^{2,2} \times \mathbb{R}^{n_{E}}+n_{I}}\left(\left(u^{\sigma}, G\left(u^{\sigma}\right)\right), \mathcal{K}_{1} \times\right.$ $\left.\mathcal{K}_{2}\right)=o(\sigma)$. By $(R C Q)$ and classical metric regularity results (see [37]) we have the existence of $\tilde{u}^{\sigma} \in \mathcal{K}$ and $o_{2,2}(\sigma) \in L^{2,2}(Q)$ such that $\left\|o_{2,2}(\sigma)\right\|_{2,2} / \sigma \rightarrow 0$ and $\tilde{u}^{\sigma}=u^{\sigma}+o_{2,2}(\sigma)$, which implies that $h \in T_{\mathcal{K}}(\bar{u})$. 
Definition 4.3. We say that $\lambda=\left(\lambda_{E}, \lambda_{I}\right) \in \mathbb{R}^{n_{E}} \times \mathbb{R}^{n_{I}}$ is a Lagrange multiplier at $\bar{u}$ if (recall (3.10))

$$
D_{u} \mathcal{L}(\bar{u}, \lambda)(v-\bar{u}) \geq 0 \text { for all } v \in \mathcal{K}_{1}, \quad \lambda_{I}^{j} \geq 0, \lambda_{I}^{j} G_{I}^{j}(\bar{u})=0, \text { for all } j=1, \ldots, n_{I} .
$$

The set of Lagrange multipliers at $\bar{u}$ is denoted as $\Lambda_{L}(\bar{u})$.

Remark 4.4. Equivalently, by Remark 3.16 and defining $\bar{p}_{\lambda}$ as the solution of (3.16),

$$
Q_{1}[\bar{u}, \lambda] v \geq 0 \text { for all } v \in T_{\mathcal{K}_{1}}(\bar{u}), \lambda_{I}^{j} \geq 0, \lambda_{I}^{j} G_{I}^{j}(\bar{u}, \lambda)=0, \text { for all } j=1, \ldots, n_{I} .
$$

Note that (4.7) implies that for a.a. $(t, x)$ we have that $\bar{H}_{u}[\lambda](t, x) v \geq 0$ for all $v \in T_{[a(t, x), b(t, x)]}(\bar{u}(t, x))$.

Under $(R C Q)$ and classical techniques for optimization problems in Banach spaces (see e.g. [11]) we have that $\Lambda_{L}(\bar{u})$ is a nonempty compact set at any weak local solution $\bar{u}$ of $(P)$. However, we point out that the result is not an immediate consequence of the abstract results in [11], because under our assumptions is not clear that the data is differentiable in $L^{2,2}(Q)$ since the perturbed states can be discontinuous. This issue can be solved by using perturbations in the $\|\cdot\|_{\infty, \infty}$ norm for the control, where the data is differentiable, and density arguments. We do not provide the details, because first order conditions are classic (see e.g. the result below) and this strategy will be followed in the next section to establish second order optimality conditions.

As shown in [16], a more precise information is provided by the following local Pontryagin minimum principle.

Theorem 4.5. Let $\bar{u}$ be a weak local solution of $(P)$. Then, there exists $\varepsilon>0$ and $\lambda \in \Lambda_{L}(\bar{u})$ such that a.e. in $Q$

$$
\bar{u}(t, x) \in \operatorname{argmin}\left\{H\left(t, x, \bar{y}(t, x), \bar{p}_{\lambda}(t, x), v\right) ; v \in[a(t, x), b(t, x)], \quad|v-\bar{u}(t, x)| \leq \varepsilon\right\} .
$$

Proof. A non-qualified local Pontryagin principle for problems involving semilinear parabolic equations with Neumann boundary conditions and mixed control-state constrains is proved in [16, Corollary 2.2]. With the obvious modifications in the proof, since we deal with Dirichlet boundary conditions and final state constraints, the non-qualified Pontryagin principle for our problem follows. The qualified result is a consequence of $(R C Q)$.

\section{$5 \quad$ Second order necessary and sufficient conditions}

Let us recall that critical cone $C_{\mathcal{K}}(\bar{u})$ to the set $\mathcal{K}$ at $\bar{u}$ is defined as

$$
C_{\mathcal{K}}(\bar{u}):=\left\{v \in T_{\mathcal{K}}(\bar{u}) ; D J(\bar{u}) v=0\right\} .
$$

In this section we establish second order necessary optimality conditions for problem $(P)$. We prove two types of results: the first one is the positivity of $\max _{\lambda \in \Lambda_{L}(\bar{u})} Q_{2}[\bar{u}, \lambda]$ on a subset of the classical critical cone, which coincides with it under a strict complementarity assumption for the finite dimensional problem (4.8). This is our main result since the assumption on the data is the same one than the main assumption for first order optimality conditions, namely the $(R C Q)$ constraint qualification condition. Under a stronger assumption, following the ideas in [13], we prove in subsection 5.2 that for a weak solution the set of Lagrange multiplier is a singleton $\{\lambda\}$ and positivity of $Q_{2}[\bar{u}, \lambda]$ is satisfied on the classical critical cone $C_{\mathcal{K}}(\bar{u})$.

In the remaining of this section $\bar{u}$ is a weak local solution, $\bar{y}=y[\bar{u}]$ and for $\lambda \in \Lambda_{L}(\bar{u})$, the adjoint state $\bar{p}_{\lambda}$ is defined by (3.16). 


\subsection{Second order necessary condition on the strict critical cone}

The strict critical cone $C_{\mathcal{K}}^{S}(\bar{u})$ to the set $\mathcal{K}$ at $\bar{u}$ is defined as (recall (4.3))

$$
C_{\mathcal{K}}^{S}(\bar{u}):=\left\{v \in C_{\mathcal{K}}(\bar{u}) ; v=0 \text { a.e. in } A_{a}(\bar{u}) \cup A_{b}(\bar{u})\right\} .
$$

Remark 5.1. Note that for every $\lambda \in \Lambda_{L}(\bar{u})$ and $v \in C_{\mathcal{K}}(\bar{u})$ we have that $\bar{H}_{u}[\lambda](t, x) v(t, x)=0$ a.e. in $Q$ (see Remark 4.4 and Lemma 5.4 below). Therefore, if there exists $\lambda \in \Lambda_{L}(\bar{u})$ such that $\bar{H}_{u}[\lambda](t, x) \neq 0$ a.e. in $A_{a}(\bar{u}) \cup A_{b}(\bar{u})$, we have that $C_{\mathcal{K}}^{S}(\bar{u})=C_{\mathcal{K}}(\bar{u})$. Note that this last assumption corresponds to the fact that there exists $\lambda \in \Lambda_{L}(\bar{u})$ such that a.e in $Q$ strict complementarity holds at the solution $\bar{u}(t, x)$ of $(4.8)$.

We now prove the following second order necessary optimality condition:

Theorem 5.2. Suppose that $(\mathbf{H 1})-(\mathbf{H 3})$ are in force. Then, for every $v \in C_{\mathcal{K}}^{S}(\bar{u})$

$$
\max _{\lambda \in \Lambda_{L}(\bar{u})} Q_{2}[\bar{u}, \lambda](v) \geq 0 .
$$

If in addition, $\bar{u}$ is a local solution satisfying the quadratic growth property (2.13), then there exists $\alpha>0$ such that for every $v \in C_{\mathcal{K}}^{S}(\bar{u})$

$$
\max _{\lambda \in \Lambda_{L}(\bar{u})} Q_{2}[\bar{u}, \lambda](v) \geq \alpha\|v\|_{2,2}^{2} .
$$

Proof. First note that the subspace of $L^{2,2}(Q)$

$$
V^{1}:=\left\{v \in L^{\infty, \infty}(Q) ; \exists \varepsilon>0 \text { such that } v(t, x)=0 \text { in } A_{a}^{\varepsilon}(\bar{u}) \cup A_{b}^{\varepsilon}(\bar{u})\right\},
$$

where

$$
\begin{aligned}
& A_{a}^{\varepsilon}(\bar{u}):=\{(t, x) \in Q ; a(t, x) \leq \bar{u}(t, x) \leq a(t, x)+\varepsilon\}, \\
& A_{b}^{\varepsilon}(\bar{u}):=\{(t, x) \in Q ; b(t, x)-\varepsilon \leq \bar{u}(t, x) \leq b(t, x)\},
\end{aligned}
$$

is dense in $V^{2}:=\left\{v \in L^{2,2}(Q) ; v=0\right.$ in $\left.A_{a}(\bar{u}) \cup A_{b}(\bar{u})\right\}$. Thus, noticing that

$$
C_{K}^{S}(\bar{u})=V^{2} \cap\left\{v \in L^{2,2}(Q) ; D J(\bar{u}) v=0, \quad D G_{E}(\bar{u}) v=0, D G_{I}^{j}(\bar{u}) v \leq 0 \forall j \in I^{1}(\bar{u})\right\},
$$

by Dmitruk's lemma (see [23, Lemma 1]) and using that $\Lambda_{L}(\bar{u})$ is compact, it is enough to prove that (5.3) holds for every

$$
v \in \hat{C}_{\mathcal{K}}^{S}(\bar{u}):=V^{1} \cap\left\{v \in L^{2,2}(Q) ; D J(\bar{u}) v=0, \quad D G_{E}(\bar{u}) v=0, \quad D G_{I}^{j}(\bar{u}) v \leq 0 \forall j \in I^{1}(\bar{u})\right\} .
$$

Given $v \in \hat{C}_{\mathcal{K}}^{S}(\bar{u})$ let us define $I^{2}(\bar{u}, v):=\left\{j \in I^{1}(\bar{u}) ; D G_{I}^{j}(\bar{u}) v=0\right\}$, and consider the conic problem

$$
\begin{gathered}
\inf _{w \in L^{\infty, \infty}(Q)} D^{2} J(\bar{u}) v^{2}+D J(\bar{u}) w \\
D^{2} G_{E}(\bar{u}) v^{2}+D G_{E}(\bar{u}) w=0, \\
\text { subjet to } \begin{aligned}
D^{2} G_{I}^{j}(\bar{u}) v^{2}+D G_{I}^{j}(\bar{u}) w \leq 0, \text { for all } j \in I^{2}(\bar{u}, v), \\
w \geq 0 \text { if } A_{a}(\bar{u}), w \leq 0 \text { in } A_{b}(\bar{u}) .
\end{aligned}
\end{gathered}
$$

First step: We prove that the value of $\left(C P_{v}\right)$ is non-negative. First, noting that

$$
R_{\mathcal{K}_{1}}(\bar{u}) \subseteq\left\{w \in L^{\infty, \infty}(Q) ; w \geq 0 \text { if } A_{a}(\bar{u}), \quad w \leq 0 \text { in } A_{b}(\bar{u})\right\},
$$


we have that $(R C Q)$ and (4.2) imply that the feasible set of $\left(C P_{v}\right)$ is non-empty. Now, let $w$ be feasible for $\left(C P_{v}\right)$ and for $\sigma>0$ set $u^{\sigma}:=\bar{u}+\sigma v+\frac{1}{2} \sigma^{2} w$. Since $v \in \hat{C}_{\mathcal{K}}^{S}(\bar{u})$ we easily check that for $\sigma$ small enough $d_{L^{1,1}(Q)}\left(u^{\sigma}, \mathcal{K}_{1}\right)=o\left(\sigma^{2}\right)$. On the other hand, we have that

$$
\begin{aligned}
& G_{E}\left(u^{\sigma}\right)=G_{E}(\bar{u})+\sigma D G_{E}(\bar{u}) v+\frac{\sigma^{2}}{2}\left[D^{2} G_{E}(\bar{u}) v^{2}+D G_{E}(\bar{u}) w\right]+\sigma^{3} O\left(\|v\|_{\infty, \infty}\|v\|_{2,2}^{2}\right)=o\left(\sigma^{2}\right), \\
& G_{I}^{j}\left(u^{\sigma}\right)=G_{I}^{j}(\bar{u})+\sigma D G_{I}^{j}(\bar{u}) v+\frac{\sigma^{2}}{2}\left[D^{2} G_{I}^{j}(\bar{u}) v^{2}+D G_{I}^{j}(\bar{u}) w\right]+\sigma^{3} O\left(\|v\|_{\infty, \infty}\|v\|_{2,2}^{2}\right),
\end{aligned}
$$

for all $j=1, \ldots, n_{I}$. Thus, if $j \notin I^{2}(\bar{u}, h)$ we get that $G_{I}^{j}\left(u^{\sigma}\right)<0$ for $\sigma$ small enough. Otherwise, if $j \in I^{2}(\bar{u}, v)$, then by the inequality constraints in $\left(C P_{v}\right)$ we obtain that $G_{I}^{j}\left(u^{\sigma}\right) \leq o\left(\sigma^{2}\right)$. Now, let us define $\hat{w}^{\sigma}:=w \mathbb{I}_{A^{\sigma}}$, where $A^{\sigma}:=\left\{(t, x) \in Q ; a-\frac{\sigma^{2}}{2} w \leq \bar{u} \leq b-\frac{\sigma^{2}}{2} w\right\}$, and set $\hat{u}^{\sigma}:=$ $\bar{u}+\sigma v+\frac{1}{2} \sigma^{2} \hat{w}^{\sigma}$. Clearly, for $\sigma$ small enough, $\hat{u}^{\sigma} \in \mathcal{K}_{1}$ and $\left\|u^{\sigma}-\hat{u}^{\sigma}\right\|_{1,1}=o\left(\sigma^{2}\right)$, which implies with (5.6) that $G_{E}\left(\hat{u}^{\sigma}\right)=G_{E}\left(u^{\sigma}\right)+o\left(\sigma^{2}\right)=o\left(\sigma^{2}\right)$ and $G_{I}^{j}\left(\hat{u}^{\sigma}\right)=G_{I}^{j}\left(u^{\sigma}\right)+o\left(\sigma^{2}\right) \leq o\left(\sigma^{2}\right)$ for all $j=1, \ldots, n_{I}$. Therefore, $d_{L^{\infty, \infty} \times \mathbb{R}^{n} E^{+n_{I}}}\left(\left(\hat{u}^{\sigma},\left(G_{E}\left(u^{\sigma}\right), G_{I}\left(u^{\sigma}\right)\right)\right) ; \mathcal{K}_{1} \times \mathcal{K}_{2}\right)=o\left(\sigma^{2}\right)$. By Robinson constraint qualification (RCQ) and classic metric regularity results (see [37] and [11, Theorem 2.87]), there exists $\tilde{u}^{\sigma} \in \mathcal{K}$ with $\left\|\tilde{u}^{\sigma}-\hat{u}^{\sigma}\right\|_{\infty, \infty}=o\left(\sigma^{2}\right)$. In particular, $\left\|\tilde{u}^{\sigma}-u^{\sigma}\right\|_{1,1}=o\left(\sigma^{2}\right)$ and so

$$
J\left(\tilde{u}^{\sigma}\right)=J\left(u^{\sigma}\right)+o\left(\sigma^{2}\right)=J(\bar{u})+\sigma D J(\bar{u}) v+\frac{\sigma^{2}}{2}\left[D^{2} J(\bar{u}) v^{2}+D J(\bar{u}) w\right]+o\left(\sigma^{2}\right) .
$$

Since $D J(\bar{u}) v=0,\left\|\tilde{u}^{\sigma}-\bar{u}\right\|_{\infty, \infty}=O\left(\sigma^{2}\right)=o(1)$ and $\tilde{u}^{\sigma}$ is feasible, dividing by $\sigma^{2}$ and using the local weak optimality of $\bar{u}$, we get

$$
0 \leq D^{2} J(\bar{u}) v^{2}+D J(\bar{u}) w+o(1),
$$

and the claim follows by letting $\sigma \downarrow 0$.

Second step: We calculate the dual problem of $\left(C P_{v}\right)$. The Lagrangian $\hat{L}: L^{\infty, \infty}(Q) \times \mathbb{R}^{n_{E}} \times \mathbb{R}^{I^{2}(\bar{u}, v)}$ associated to problem $\left(C P_{v}\right)$ is defined as

$$
\begin{aligned}
\hat{L}\left(w, \lambda_{E}, \hat{\lambda}_{I}\right):= & D^{2} J(\bar{u}) v^{2}+D J(\bar{u}) w+\lambda_{E}^{\top}\left[D^{2} G_{E}(\bar{u}) v^{2}+D G_{E}(\bar{u}) w\right] \\
& +\sum_{j \in I^{2}(\bar{u}, v)} \hat{\lambda}_{I}^{j}\left[D^{2} G_{I}^{j}(\bar{u}) v^{2}+D G_{I}^{j}(\bar{u}) w\right], \\
= & D_{u u}^{2} \mathcal{L}\left(\bar{u}, \lambda_{E}, \lambda_{I}\right) v^{2}+D_{u} \mathcal{L}\left(\bar{u}, \lambda_{E}, \lambda_{I}\right) w
\end{aligned}
$$

where $\lambda_{I} \in \mathbb{R}_{+}^{n_{I}}$ is defined as $\lambda_{I}^{j}=\hat{\lambda}_{I}^{j}$ if $j \in I^{2}(\bar{u}, v)$ and 0 , otherwise. Therefore, the dual problem of $\left(C P_{v}\right)$ is

$$
\max _{\left(\lambda_{E}, \hat{\lambda}_{I}\right) \in \mathbb{R}^{n_{E}} \times \mathbb{R}_{+}^{I^{2}(\bar{u}, v)}}\left\{D_{u u}^{2} \mathcal{L}\left(\bar{u}, \lambda_{E}, \lambda_{I}\right) v^{2}+\inf _{w \in T_{\mathcal{K}_{1}}(\bar{u}) \cap L^{\infty, \infty}(Q)} D_{u} \mathcal{L}\left(\bar{u}, \lambda_{E}, \lambda_{I}\right) w\right\} .
$$

Now, noting that $v \in C_{\mathcal{K}}(\bar{u})$ implies that for every $\left(\lambda_{E}, \lambda_{I}\right) \in \Lambda_{L}(\bar{u})$ we have that $\lambda_{I}^{i}=0$ if $i \in\left\{1, \ldots, n_{I}\right\} \backslash I^{2}(\bar{u}, v)$, we get that the dual problem can be written as

$$
\max _{\left(\lambda_{E}, \lambda_{I}\right) \in \Lambda_{L}(\bar{u})}\left\{D_{u u}^{2} \mathcal{L}\left(\bar{u}, \lambda_{E}, \lambda_{I}\right) v^{2}+\inf _{w \in T_{\mathcal{K}_{1}}(\bar{u}) \cap L^{\infty, \infty}(Q)} D_{u} \mathcal{L}\left(\bar{u}, \lambda_{E}, \lambda_{I}\right) w\right\} .
$$

But since

$$
\inf _{w \in T_{\mathcal{K}_{1}}(\bar{u}) \cap L^{\infty, \infty}(Q)} D_{u} \mathcal{L}\left(\bar{u}, \lambda_{E}, \lambda_{I}\right) w=\left\{\begin{array}{l}
0 \text { if }\left(\lambda_{E}, \lambda_{I}\right) \in \Lambda_{L}(\bar{u}), \\
-\infty \text { if not }
\end{array}\right.
$$

we obtain that the dual problem is given by

$$
\max _{\left(\lambda_{E}, \lambda_{I}\right) \in \Lambda_{L}(\bar{u})} D_{u u}^{2} \mathcal{L}\left(\bar{u}, \lambda_{E}, \lambda_{I}\right) v^{2}=\max _{\lambda \in \Lambda_{L}(\bar{u})} Q_{2}[\bar{u}, \lambda](v),
$$


by Proposition 3.5 .

Third step: We prove that $\left(C P_{v}\right)$ and its dual have the same value. Let $\hat{K}_{1}:=T_{\mathcal{K}_{1}}(\bar{u}) \cap L^{\infty, \infty}(Q)$, $\hat{K}_{2}:=\{0\}^{n_{E}} \times \mathbb{R}_{-}^{I^{2}(\bar{u}, v)}$ and $\hat{G}:=\left(G_{E},\left(G_{I}^{i}\right)_{i \in I^{2}(\bar{u}, v)}\right)$. Then, the constraints for $\left(C P_{v}\right)$ can be written in compact form

$$
D \hat{G}(\bar{u}) w+D^{2} \hat{G}(\bar{u}) v^{2} \in \hat{K}_{2}, w \in \hat{K}_{1} .
$$

Since $R_{\mathcal{K}_{1}}(\bar{u}) \subseteq \hat{K}_{1}$ and $\hat{K}_{2}$ is the projection of $R_{\mathcal{K}_{2}}(G(\bar{u}))$ on $\{0\}^{n_{E}} \times \mathbb{R}_{-}^{I^{2}(\bar{u}, v)}$, by $(R C Q)$ and (4.2) we have that

$$
0 \in \operatorname{int}\left\{D \hat{G}(\bar{u}) \hat{K}_{1}+D^{2} \hat{G}(\bar{u}) v^{2}-\hat{K}_{2}\right\} .
$$

Therefore, by [11, Theorem 2.187] we have that the value of $\left(C P_{v}\right)$ is equal to the value of its dual and so (5.3) follows.

Fourth step: We prove (5.4). Defining $J_{\alpha}(u):=J(u)-\frac{1}{2} \alpha\|u-\bar{u}\|_{2,2}^{2},(2.13)$ implies that $\bar{u}$ is a weak local solution of the problem $\left(P_{\alpha}\right)$ defined as $\inf \left\{J_{\alpha}(u) ; u \in \mathcal{K}\right\}$. Since $D J_{\alpha}(u)=D J(u)-\alpha(u-\bar{u})$, the strict critical cone at $\bar{u}$ associated to $\left(P_{\alpha}\right)$ coincides with $C_{\mathcal{K}}^{S}(\bar{u})$. Moreover, the Lagrangian $\mathcal{L}_{\alpha}(u, \lambda):=\mathcal{L}(u, \lambda)-\frac{1}{2} \alpha\|u-\bar{u}\|_{2,2}^{2}$, associated to $\left(P_{\alpha}\right)$, satisfies $D_{u u}^{2} \mathcal{L}_{\alpha}(u, \lambda) v^{2}=D_{u u}^{2} \mathcal{L}(u, \lambda) v^{2}-$ $\alpha\|v\|_{2,2}^{2}$. Condition (5.4) follows from (5.3) applied to the problem $\left(P_{\alpha}\right)$.

\subsection{Second order necessary conditions using polyhedricity theory}

Given $\lambda:=\left(\lambda_{E}, \lambda_{I}\right) \in \Lambda_{L}(\bar{u})$ define $\bar{q}_{\lambda}(t, x):=-\bar{H}_{u}[\lambda](t, x)$ for a.a. $(t, x) \in Q$. Note that $\bar{q}_{\lambda} \in$ $L^{\infty, \infty}(Q)$. Let us set $\left(\bar{q}_{\lambda}\right)^{\perp}:=\left\{v \in L^{2,2}(Q) ;\left\langle\bar{q}_{\lambda}, v\right\rangle_{L^{2}}=0\right\}$. In this section, we will assume the following hypothesis

(H4) There exists $\lambda \in \Lambda_{L}(\bar{u})$ such that

$$
0 \in \operatorname{int}\left(D G(\bar{u})\left[\left(\mathcal{K}_{1}-\bar{u}\right) \cap\left(\bar{q}_{\lambda}\right)^{\perp}\right]-T_{\mathcal{K}_{2}}(G(\bar{u})) \cap \lambda^{\perp}\right)
$$

where $\lambda^{\perp}$ denotes the subspace of $\mathbb{R}^{n_{E}} \times \mathbb{R}^{n_{I}}$ which is orthogonal to $\lambda$.

Remark 5.3. (i) Clearly, (5.8) implies (RCQ).

(ii) On the other hand, recalling the definition of $A_{a}^{\varepsilon}(\bar{u})$ and $A_{b}^{\varepsilon}(\bar{u})$ in (5.5), condition (5.8) is implied by the existence of $\varepsilon>0$ and $v_{E, \varepsilon}^{i}\left(i=1, \ldots, n_{E}\right), v_{I, \varepsilon}^{j}\left(j \in I^{1}(\bar{u})\right)$ such that $\operatorname{supp}\left(v_{E, \varepsilon}^{i}\right) \subseteq$ $\left(A_{a}^{\varepsilon}(\bar{u}) \cup A_{b}^{\varepsilon}\right)^{c}, \operatorname{supp}\left(v_{I, \varepsilon}^{j}\right) \subseteq\left(A_{a}^{\varepsilon}(\bar{u}) \cup A_{b}^{\varepsilon}\right)^{c}$ and

$$
D G_{E}^{i}(\bar{u}) v_{E, \varepsilon}^{k}=\delta_{i k} \quad D G_{I}^{j}(\bar{u}) v_{I, \varepsilon}^{k}=\delta_{j k},
$$

where $\delta_{i k}$ is the Kronecker symbol. This type of surjectivity condition is the usual assumption in the existing literature [7, 15, 17, 18].

The following Lemma will be useful.

Lemma 5.4. Let $\lambda \in \Lambda_{L}(\bar{u})$. Then, the following assertions hold true:

(i) The critical cone can be expressed as

$$
C_{\mathcal{K}}(\bar{u}):=\left\{v \in T_{\mathcal{K}_{1}}(\bar{u}) \cap\left(\bar{q}_{\lambda}\right)^{\perp} ; D G(\bar{u}) v \in T_{\mathcal{K}_{2}}(G(\bar{u})) \cap \lambda^{\perp}\right\} .
$$

(ii) If (H4) holds and $\lambda_{1} \in \Lambda_{L}(\bar{u})$, then $\lambda_{1}=\lambda$. 
Proof. Since $\lambda \in \Lambda_{L}(\bar{u})$, for any $v \in C_{\mathcal{K}}(\bar{u})$, we have

$$
D_{u} \mathcal{L}(\bar{u}, \lambda) v=D J(\bar{u}) v+\langle\lambda, D G(\bar{u}) v\rangle=Q_{1}[\bar{u}, \lambda] v=-\int_{Q} \bar{q}_{\lambda}(t, x) v(t, x) \mathrm{d} t \mathrm{~d} x \geq 0 .
$$

Since for all $j \in I^{1}(\bar{u}), D G_{I}^{j}(\bar{u}) v \leq 0, \lambda_{I}^{j} \geq 0$ and $\lambda_{I}^{j}=0$ for $j \in\left\{1, \ldots, n_{I}\right\} \backslash I^{1}(\bar{u})$, we get

$$
0 \geq \sum_{j \in I^{1}(\bar{u})} \lambda^{j} D G_{I}^{j}(\bar{u}) v=-\int_{Q} \bar{q}_{\lambda}(t, x) v(t, x) \mathrm{d} t \mathrm{~d} x \geq 0,
$$

which implies that $v \in\left(\bar{q}_{\lambda}\right)^{\perp}$ and $D G(\bar{u}) v \in \lambda^{\perp}$. Conversely, if $v \in\left(\bar{q}_{\lambda}\right)^{\perp}$ and $D G(\bar{u}) v \in \lambda^{\perp}$, the fact that

$$
D J(\bar{u}) v+\langle\lambda, D G(\bar{u}) v\rangle=-\int_{Q} \bar{q}_{\lambda}(t, x) v(t, x) \mathrm{d} t \mathrm{~d} x \text { for all } v \in L^{2,2}(Q),
$$

implies that $D J(\bar{u}) v=0$. Assertion (i) follows. Now, given $\delta \in \mathbb{R}^{n_{E}+n_{I}}$, with $|\delta|$ small enough, (5.8) implies the existence of $v_{\delta} \in \mathcal{K}_{1}-\bar{u}$ and $z \in T_{\mathcal{K}_{2}}(G(\bar{u}))$ such that

$$
D G(\bar{u}) v_{\delta}=\delta+z, \quad\left\langle\bar{q}_{\lambda}, v_{\delta}\right\rangle_{L^{2}}=0, \quad\langle\lambda, z\rangle=0 .
$$

If $\lambda_{1} \in \Lambda_{L}(\bar{u})$, then

$$
D J(\bar{u})+\bar{q}_{\lambda_{1}}+D G(\bar{u})^{*} \lambda_{1}=0, \quad \lambda_{1} \in N_{\mathcal{K}_{2}}(G(\bar{u})), \quad \bar{q}_{\lambda_{1}} \in N_{\mathcal{K}_{1}}(\bar{u}) .
$$

Thus, since $\lambda \in \Lambda_{L}(\bar{u})$, we obtain that

$$
\left\langle\bar{q}_{\lambda_{1}}, v_{\delta}\right\rangle_{L^{2}}+\left\langle\lambda_{1}, \delta+z\right\rangle=\left\langle\bar{q}_{\lambda}, v_{\delta}\right\rangle_{L^{2}}+\langle\lambda, \delta+z\rangle=\langle\lambda, \delta\rangle
$$

from which

$$
\left\langle\lambda-\lambda_{1}, \delta\right\rangle=\left\langle\bar{q}_{\lambda_{1}}, v_{\delta}\right\rangle_{L^{2}}+\left\langle\lambda_{1}, z\right\rangle \leq 0
$$

Since $\delta$ is arbitrary but small, the above relation implies that $\lambda=\lambda_{1}$. The result follows.

Definition 5.5. Let $\left(X,\|\cdot\|_{X}\right)$ be a Banach space and let $K \subseteq X$ be a non-empty, closed and convex set. We say that $K$ is polyhedric at $x \in K$ if for every $q \in N_{K}(x)$ we have that $\operatorname{clos}_{X}\left(R_{K}(x) \cap\right.$ $\operatorname{Ker}(q))=T_{K}(x) \cap \operatorname{Ker}(q)$. If $K$ is polyhedric at every $x \in K$ we will say that $K$ is polyhedric.

We have the following density results regarding the critical cone.

Lemma 5.6. The following assertions hold true:

(i) The set $K_{1}$ is polyhedric.

(ii) If (5.8) holds at $\bar{u}$, then $\operatorname{clos}_{L^{2,2}}\left(R_{\mathcal{K}_{1}}(\bar{u}) \cap C_{\mathcal{K}}(\bar{u})\right)=C_{\mathcal{K}}(\bar{u})$.

Proof. Let $q \in N_{\mathcal{K}_{1}}(\bar{u})$ and $v \in T_{\mathcal{K}_{1}}(\bar{u}) \cap q^{\perp}$. For $k \in \mathbb{N} \backslash\{0\}$ set

$$
v_{k}(t, x):=\left\{\begin{aligned}
v(t, x) & \text { if } \bar{u}(t, x)+\frac{1}{k} v(t, x) \in[a(t, x), b(t, x)], \\
0 & \text { otherwise. }
\end{aligned}\right.
$$

Clearly, $v_{k} \in R_{\mathcal{K}_{1}}(u) \cap q^{\perp}$. Moreover, since $v \in T_{\mathcal{K}_{1}}(\bar{u})$, as $k \uparrow \infty$ we have that $v_{k} \rightarrow v$ in $L^{2,2}(Q)$ by dominated convergence. Assertion (i) follows. Now, suppose that (5.8) holds at $\bar{u}$ and let $v \in C_{\mathcal{K}}(\bar{u})$. By Lemma 5.4 and (i) we have the existence of $\lambda \in \mathbb{R}^{n_{E}+n_{I}}$ and $v_{\varepsilon}^{1} \in R_{\mathcal{K}_{1}}(\bar{u}) \cap\left(\bar{q}_{\lambda}\right)^{\perp}$ such that $\Lambda_{L}(\bar{u})=\{\lambda\}$ and $v_{\varepsilon}^{1}=v+o(1)$ in $L^{2,2}(Q)$, as $\varepsilon \downarrow 0$. Therefore, for $\varepsilon>0$ small enough $\varepsilon v_{\varepsilon}^{1} \in$ $\left(\mathcal{K}_{1}-\bar{u}\right) \cap\left(\bar{q}_{\lambda}\right)^{\perp}$. Moreover, 


$$
\lambda^{\top} D G(\bar{u})\left(\varepsilon v_{\varepsilon}^{1}\right)=o(\varepsilon), \quad D G_{E}(\bar{u})\left(\varepsilon v_{\varepsilon}^{1}\right)=o(\varepsilon), \quad D G_{I}^{j}(\bar{u})\left(\varepsilon v_{\varepsilon}^{1}\right) \leq o(\varepsilon),
$$

for all $j \in I^{1}(\bar{u})$. Thus,

$$
d_{L^{\infty}, \infty}(Q) \times \mathbb{R}^{n_{E} E^{+n_{I}}}\left(\left(\varepsilon v_{\varepsilon}^{1}, D G(\bar{u})\left(\varepsilon v_{\varepsilon}^{1}\right)\right),\left[\left(\mathcal{K}_{1}-\bar{u}\right) \cap\left(\bar{q}_{\lambda}\right)^{\perp}\right] \times\left[T_{\mathcal{K}_{2}}(G(\bar{u})) \cap \lambda^{\perp}\right]\right)=o(\varepsilon),
$$

which implies, by the regularity condition (5.8), the existence of $v_{\varepsilon}^{2} \in\left(\mathcal{K}_{1}-\bar{u}\right) \cap\left(q_{\lambda}\right)^{\perp}$ such that $D G(\bar{u}) v_{\varepsilon}^{2} \in T_{\mathcal{K}_{2}}(G(\bar{u})) \cap \lambda^{\perp}$ and $v_{\varepsilon}^{2}=\varepsilon v_{\varepsilon}^{1}+o(\varepsilon)$ in $L^{\infty, \infty}(Q)$. Therefore, $\frac{1}{\varepsilon} v_{\varepsilon}^{2} \in R_{\mathcal{K}_{1}}(\bar{u}) \cap C_{\mathcal{K}}(\bar{u})$ and $\frac{1}{\varepsilon} v_{\varepsilon}^{2}=v+o(1)$ in $L^{2,2}(Q)$. The result follows.

Now we can prove second order optimality conditions in the critical cone $C_{\mathcal{K}}(\bar{u})$ assuming the stronger assumption (5.8).

Theorem 5.7. Let $\bar{u}$ be a local solution of $(P)$ and suppose that $(\mathbf{H 4})$ holds at $\bar{u}$. Then, the set of Lagrange multipliers $\Lambda_{L}(\bar{u})$ is a singleton $\lambda_{L}(\bar{u})=\{\lambda\}$ and the following second order necessary condition holds true:

$$
Q_{2}[\bar{u}, \lambda](v) \geq 0 \text { for all } v \in C_{\mathcal{K}}(\bar{u}) .
$$

If in addition, $\bar{u}$ is a local solution satisfying the quadratic growth property (2.13), then there exists $\alpha>0$ such that for every $v \in C_{\mathcal{K}}(\bar{u})$

$$
Q_{2}[\bar{u}, \lambda](v) \geq \alpha\|v\|_{2,2}^{2} .
$$

Proof. We follow the same strategy used in the proof of Theorem 5.2. By Lemma 5.6 (ii) and the continuity in $L^{2,2}(Q)$ of $D^{2} \mathcal{L}(\bar{u}, \lambda)(\cdot, \cdot)$, it suffices to prove (5.10) for critical directions in $R_{\mathcal{K}_{1}}(\bar{u}) \cap$ $C_{\mathcal{K}}(\bar{u})$. Let us fix $v \in R_{\mathcal{K}_{1}}(\bar{u}) \cap C_{\mathcal{K}}(\bar{u})$. If we prove that the value of $\left(C P_{v}\right)$, defined in the first step in the proof of Theorem 5.2, is non-negative, we are done because the proofs of the second, third and fourth steps remain unchanged and the Lagrange multiplier $\lambda$ is unique by Lemma 5.4(ii). Let $w$ be feasible for $\left(C P_{v}\right)$ and for $\sigma>0$ set $u^{\sigma}:=\bar{u}+\sigma v+\frac{1}{2} \sigma^{2} w$. Using that $v \in R_{\mathcal{K}_{1}}(\bar{u})$ we have that $\bar{u}+\sigma v \in \mathcal{K}_{1}$, for $\sigma$ small enough. This implies that $d_{L^{1,1}(Q)}\left(u^{\sigma}, \mathcal{K}_{1}\right)=o\left(\sigma^{2}\right)$, as $\sigma \downarrow 0$. As in the proof in Theorem 5.2, defining $\hat{w}^{\sigma}:=w \mathbb{I}_{A^{\sigma}}$, where $A^{\sigma}:=\left\{(t, x) \in Q ; a-\frac{\sigma^{2}}{2} w \leq \bar{u} \leq b-\frac{\sigma^{2}}{2} w\right\}$, and setting $\hat{u}^{\sigma}:=\bar{u}+\sigma v+\frac{1}{2} \sigma^{2} \hat{w}^{\sigma}$, we obtain that $d_{L^{\infty, \infty}(Q) \times \mathbb{R}^{n_{E}+n_{I}}}\left(\left(\hat{u}^{\sigma},\left(G_{E}\left(u^{\sigma}\right), G_{I}\left(u^{\sigma}\right)\right)\right) ; \mathcal{K}_{1} \times \mathcal{K}_{2}\right)=o\left(\sigma^{2}\right)$. By Remark 5.3, we can mimic the proof in Theorem 5.2 in order to construct $\tilde{u}^{\sigma}$, feasible for problem $(P)$, such that $\left\|\tilde{u}^{\sigma}-\bar{u}\right\|_{\infty, \infty}=o(1)$ and $\left\|\tilde{u}^{\sigma}-u^{\sigma}\right\|_{1,1}=o\left(\sigma^{2}\right)$, from which the result easily follows.

\subsection{Second order sufficient condition}

Let us first recall the notion of Legendre form (see e.g. [30]). Given a Hilbert space $\mathcal{H}$, a quadratic form $Q: \mathcal{H} \rightarrow \mathbb{R}$ is a Legendre form if it satisfies:

(i) $Q$ is weakly lower semicontinuous.

(ii) If $v_{k} \in \mathcal{H}$ converges weakly to $\bar{v}$ and $Q\left(v_{k}\right)$ converges to $Q(\bar{v})$, then $v_{k}$ converges strongly to $\bar{v}$.

We have the following second order sufficient condition:

Theorem 5.8. Let $\bar{u} \in \mathcal{K}$. Suppose that

(i) $\Lambda_{L}(\bar{u}) \neq \emptyset$.

(ii) For all $\lambda \in \Lambda_{L}(\bar{u})$ we have that $Q_{2}[\bar{u}, \lambda]$ is a Legendre form in $L^{2,2}(Q)$.

(iii) There exists $\alpha>0$ such that for every $v \in C_{\mathcal{K}}(\bar{u})$

$$
\max _{\lambda \in \Lambda(\bar{u})} Q_{2}[\bar{u}, \lambda] v \geq \alpha\|v\|_{2,2}^{2}
$$

Then $\bar{u}$ is a weak local solution of $(P)$ satisfying the quadratic growth property (2.13). 
Proof. The proof is by contradiction. If (2.13) does not holds, then there exists a sequence $u_{k} \in \mathcal{K}$ with $\left\|u_{k}-\bar{u}\right\|_{\infty, \infty} \rightarrow 0$ and such that for all $\lambda \in \Lambda_{L}(\bar{u})$ we have

$$
\mathcal{L}\left(u_{k}, \lambda\right)-\mathcal{L}(\bar{u}, \lambda) \leq J\left(u_{k}\right)-J(\bar{u}) \leq o\left(\left\|u_{k}-\bar{u}\right\|_{2,2}^{2}\right) .
$$

Setting $\delta_{k} u:=u_{k}-\bar{u}$ and using Proposition 3.5 we obtain that

$$
Q_{1}[\bar{u}, \lambda] \delta_{k} u+\frac{1}{2} Q_{2}[\bar{u}, \lambda]\left(\delta_{k} u\right) \leq o\left(\left\|\delta_{k} u\right\|_{2,2}^{2}\right) .
$$

Defining $\tau_{k}:=\left\|\delta_{k} u\right\|_{2,2}$ and $h_{k}:=\delta_{k} u / \tau_{k}$ we get that $\left\|h_{k}\right\|_{2,2}=1$. Therefore, by weak compactness, there exists $h \in L^{2,2}(Q)$ such that, except for some subsequence, $h_{k} \rightarrow h$ weakly in $L^{2,2}(Q)$. Let us prove that $h \in C_{\mathcal{K}}(\bar{u})$. Since $h_{k} \in R_{\mathcal{K}_{1}}(\bar{u})$ and $T_{\mathcal{K}_{1}}(\bar{u})$ is weakly closed, we get that $h \in T_{\mathcal{K}_{1}}(\bar{u})$. On the other hand, using the expansion in Proposition 3.3, we get

$$
\begin{aligned}
0=G_{E}^{i}\left(u_{k}\right) & =G_{E}^{i}(\bar{u})+\tau_{k} D G_{E}^{i}(\bar{u}) h_{k}+O\left(\tau_{k}^{2}\right)=\tau_{k} \int_{\Omega}\left(\Phi_{E}^{i}\right)_{y}(x, \bar{y}(T, x)) z\left[h_{k}\right](T, x) \mathrm{d} x+O\left(\tau_{k}^{2}\right), \\
0 \geq G_{I}^{j}\left(u_{k}\right) & =G_{I}^{j}(\bar{u})+\tau_{k} D G_{I}^{j}(\bar{u}) h_{k}+O\left(\tau_{k}^{2}\right)=\tau_{k} \int_{\Omega}\left(\Phi_{I}^{j}\right)_{y}(x, \bar{y}(T, x)) z\left[h_{k}\right](T, x) \mathrm{d} x+O\left(\tau_{k}^{2}\right),
\end{aligned}
$$

for all $i=1, \ldots, n_{E}, j \in I^{1}(\bar{u})$. Dividing by $\tau_{k}$ and letting $k \uparrow \infty$, Proposition 2.1(iii) implies that

$$
D G_{E}^{i}(\bar{u}) h=0 \quad \forall i=1, \ldots, n_{E} \text { and } D G_{I}^{j}(\bar{u}) h \leq 0, \quad \forall j \in I^{1}(\bar{u}) .
$$

Analogously, by Proposition 3.5 and (5.13) we have that

$$
\tau_{k} D J(\bar{u}) h_{k}+O\left(\tau_{k}^{2}\right)=J\left(u_{k}\right)-J(\bar{u}) \leq o\left(\tau_{k}^{2}\right) .
$$

Dividing by $\tau_{k}$ and letting $k \uparrow \infty$, the first identity in (3.14) with $\lambda=0$ and Proposition 2.1(iii) imply that $D J(\bar{u}) h \leq 0$. Since $h \in T_{\mathcal{K}_{1}}(\bar{u})$,

$$
D_{u} \mathcal{L}(\bar{u}, \lambda) h=D J(\bar{u}) h+\sum_{i=1}^{n_{E}} \lambda_{E}^{i} D G_{E}^{i}(\bar{u}) h+\sum_{j \in I(\bar{u})} \lambda_{I}^{j} D G_{I}^{j}(\bar{u}) h \geq 0 .
$$

Since $D G_{E}^{i}(\bar{u}) h=0$ for all $i=1, \ldots, n_{E}$ and $D G_{I}^{j}(\bar{u}) h \leq 0$ for all $j=1, \ldots, n_{I}$, we have that $D J(\bar{u}) h \geq 0$ and so $D J(\bar{u}) h=0$, which implies that $h \in C_{\mathcal{K}}(\bar{u})$. On the other hand, since $\delta_{k} u \in$ $T_{\mathcal{K}_{1}}(\bar{u})$ we have that $Q_{1}[\bar{u}, \lambda]\left(\delta_{k} u\right) \geq 0$. Thus, dividing by $\tau_{k}$ in (5.14) and using that $Q_{2}[\bar{u}, \lambda]$ is weakly lower semicontinuous, we obtain that

$$
Q_{2}[\bar{u}, \lambda](h) \leq \liminf _{k \rightarrow \infty} Q_{2}[\bar{u}, \lambda]\left(h_{k}\right) \leq \limsup _{k \rightarrow \infty} Q_{2}[\bar{u}, \lambda]\left(h_{k}\right) \leq 0 .
$$

Since $h \in C_{\mathcal{K}}(\bar{u})$ and the above identity holds for every $\lambda \in \Lambda_{L}(\bar{u})$, by $(5.12)$ we can choose $\lambda_{h} \in \Lambda_{L}(\bar{u})$ such that

$$
Q_{2}\left[\bar{u}, \lambda_{h}\right](h) \geq \alpha\|h\|_{2,2}^{2} .
$$

Therefore, (5.15) implies that $h=0$ and $Q_{2}[\bar{u}, \lambda]\left(h_{k}\right)$ converges to $0=Q_{2}[\bar{u}, \lambda](h)$. Since $Q_{2}[\bar{u}, \lambda]$ is a Legendre form, we obtain that $h_{k} \rightarrow 0$ strongly in $L^{2,2}(Q)$, which contradicts $\left\|h_{k}\right\|_{2,2}=1$. 


\section{The case of a quadratic Hamiltonian}

In this final section we sketch some particular features in the case when $\ell$ is quadratic w.r.t. $(y, u)$ and $\varphi$ is linear w.r.t. $u$. We consider as model problem the following

$$
\begin{aligned}
& \inf J(u):=\int_{Q}\left[\frac{1}{2} u(t, x)^{2}+\ell(t, x, y(t, x))\right] \mathrm{d} t \mathrm{~d} x+\int_{\Omega} \Phi(x, y(T, x)) \mathrm{d} x, \\
& \partial_{t} y-\Delta y+\varphi(y)=u \text { in } Q, \\
& \text { subject to } \\
& y=0 \text { in } \Sigma \text {, } \\
& y(0, \cdot)=y_{0} \text { in } \Omega \\
& a(t, x) \leq u(t, x) \leq b(t, x) \text { in } Q, \\
& \int_{\Omega} \Phi_{E}^{i}(x, y(T, x)) \mathrm{d} x=0 \quad \text { for } i=1, \ldots, n_{E}, \\
& \int_{\Omega} \Phi_{I}^{j}(x, y(T, x)) \mathrm{d} x \leq 0 \quad \text { for } j=1, \ldots, n_{I},
\end{aligned}
$$

where $a, b$ and $y_{0}$ are as in the previous sections and $\ell, \varphi, \Phi, \Phi_{E}^{i}, \Phi_{I}^{j}$ satisfy the assumptions in Section 2. Let $\bar{u}$ be a weak local solution of $\left(P_{1}\right)$ and suppose that $(R C Q)$ is verified at $\bar{u}$. Then, the set of Lagrange multipliers is non-empty and by Theorem 5.2 the second order condition (5.3) holds true.

On the other hand, by the proof of Proposition 3.3, the Sobolev embeddings and the particular forms of the cost and the dynamics, for $s$ large enough the residual term in the expansion of the Lagrangian can be improved to $o\left(\|v\|_{2,2}^{2}\right)$ as $\|v\|_{s, s} \rightarrow 0$ (see [5, Lemma 4.1] for detailed computations). Moreover, using Proposition 2.1(iii), it is easy to see that for all $\lambda$ we have that $Q_{2}[\bar{u}, \lambda]$ is a Legendre form. Thus, by the proof of the sufficient condition in Theorem 5.8, if (5.12) is verified then the quadratic growth property (2.13) is satisfied for all $u \in \mathcal{K}$ such that $\|u-\bar{u}\|_{s, s}$ is small. Therefore, Remark 2.5 implies the following result

Theorem 6.1. Consider problem $\left(P_{1}\right)$ and let $\bar{u} \in \mathcal{K}$ be such that $\Lambda_{L}(\bar{u}) \neq \emptyset$ and (5.12) are verified. Then, $\bar{u}$ satisfies the $L^{1,1}$-weak quadratic growth condition (2.14).

As a consequence we have the following corollary

Corollary 6.2. Suppose that at $\bar{u}$ either (i) $(R C Q)$ and $C_{\mathcal{K}}(\bar{u})=C_{\mathcal{K}}^{S}(\bar{u})$ or (ii) the regularity condition (5.8) are satisfied. Then, if $\bar{u}$ is a weak local solution of $\left(P_{1}\right)$ satisfying the weak quadratic growth condition (2.13), we have that $\bar{u}$ satisfies the $L^{1,1}$-weak quadratic growth condition (2.14).

Proof. If $\bar{u}$ is a weak local solution of $\left(P_{1}\right)$ satisfying the weak quadratic growth condition (2.13) then $\Lambda_{L}(\bar{u}) \neq \emptyset$. Moreover, if (i) or (ii) are verified, Theorem 5.2 or Theorem 5.7 implies that (5.12) is satisfied. The result follows from Theorem 6.1.

Remark 6.3. If one of the conditions in the above Corollary is satisfied then the notions of weak quadratic growth and $L^{1,1}$-weak quadratic growth coincide. Of course, when no final state constraints are imposed none of these conditions are required.

We end this section sketching how our assumptions have to be modified if $\mathcal{K}$ is not bounded in $L^{\infty, \infty}(Q)$ (the model example is when $\mathcal{K}_{1}=\left\{u \in L^{2,2}(Q) ; u(t, x) \geq 0\right.$ for a.a. $\left.(t, x) \in Q\right\}$ ). Let us assume that $u \in L^{\infty, \infty}(Q)$. In some particular cases, for example under additional restrictions over $\varphi$ such as a uniform Lipschitz property, the existence of such a solution can be ensured by proving first the existence in $L^{2,2}(Q)$ and then using a bootstrapping argument and the first order optimality conditions. Since in order to obtain second order optimality conditions the strategy is based on considering expansions of the Lagrangian when $L^{\infty, \infty}$ perturbations are performed and then some 
density arguments using the continuity of $Q_{2}[\bar{u}, \lambda]$, the only modification needed for the proof of Theorem 5.2 is that $(R C Q)$ has to be strengthened to

$$
0 \in \operatorname{int}\left\{G(\bar{u})+D G(\bar{u})\left(\mathcal{K}_{1} \cap L^{\infty, \infty}(Q)-\bar{u}\right)-\mathcal{K}_{2}\right\} .
$$

Similarly, the corresponding modification to (5.8), which is needed to prove Theorem 5.7, is

$$
0 \in \operatorname{int}\left(D G(\bar{u})\left[\left(\mathcal{K}_{1} \cap L^{\infty, \infty}(Q)-\bar{u}\right) \cap\left(\bar{q}_{\lambda}\right)^{\perp}\right]-T_{\mathcal{K}_{2}}(G(\bar{u})) \cap \lambda^{\perp}\right) .
$$

Indeed, under (6.1) it is possible to show that $\cos _{L^{2,2}}\left(R_{\mathcal{K}_{1}}(\bar{u}) \cap L^{\infty, \infty}(Q) \cap C_{\mathcal{K}}(\bar{u})\right)=C_{\mathcal{K}}(\bar{u})$ and the proof of Theorem 5.7 remains unchanged. Thus, if either (i') $\left(R C Q^{\prime}\right)$ and $C_{\mathcal{K}}(\bar{u})=C_{\mathcal{K}}^{S}(\bar{u})$ or (ii') the regularity condition (6.1) is satisfied, we have that any weak solution $\bar{u} \in L^{\infty, \infty}(Q)$ that satisfies the weak quadratic growth condition also satisfies the $L^{s, s}$-weak quadratic growth condition if $s$ is large enough. The latter condition implies of course that if we fix $R>0$, the $L^{1,1}$-weak quadratic growth condition (2.14) holds true for every $u$ such that $\|u-\bar{u}\|_{1,1} \leq \varepsilon$ and $\|u\|_{\infty, \infty} \leq R$.

\section{References}

[1] R.A. Adams. Sobolev spaces. Academic Press, New York, 1975.

[2] J.P. Aubin. Un théorème de compacité. CRAS, 256:5042-5044, 1963.

[3] T. Bayen, J.F. Bonnans, and F. J. Silva. Characterization of local quadratic growth for strong minima in the optimal control of semi-linear elliptic equations. Trans. Amer. Math. Soc., 3664:2063-2087, 2014.

[4] E. Di Benedetto. On the local behavior of solutions of degenerate parabolic equations with measurable coefficients. Ann. Scuola Norm. Sup. Pisa CI. Sci, 13:487-535, 1986.

[5] M. Bergounioux and N. Merabet. Sensitivity analysis for optimal control of problems governed by semilinear parabolic equations. Control Cybernet., 29(4):861-886, 2000.

[6] J. F. Bonnans. Second order analysis for control constrained optimal control problems of semilinear elliptic systems. Appl. Math. Optim., 38-3:303-325, 1998.

[7] J. F. Bonnans and E. Casas. Contrôle de systèmes elliptiques semilinéaires comportant des contraintes sur l'état. In Nonlinear partial differential equations and their applications. Collège de France seminar, Vol. VIII (Paris, 1984-1985), volume 166 of Pitman Res. Notes Math. Ser., pages 69-86. Longman Sci. Tech., Harlow, 1988.

[8] J. F. Bonnans, X. Dupuis, and L. Pfeiffer. Second-order necessary conditions in Pontryagin form for optimal control problems. SIAM J. Control Optim., to appear., 2013.

[9] J.F. Bonnans and P. Jaisson. Optimal control of a time-dependent state constrained parabolic equation. SIAM J. Control Optim., 48-7:4550-4571, 2010.

[10] J.F. Bonnans and N.P. Osmolovskiü. Second-order analysis of optimal control problems with control and initial-final state constraints. Journal of Convex Analysis, 17(3):885-913, 2010.

[11] J.F. Bonnans and A. Shapiro. Perturbation analysis of optimization problems. Springer-Verlag, New York, 2000. 
[12] J.F. Bonnans and F.J. Silva. Asymptotic expansion for the solutions of control constrained semilinear elliptic problems with interior penalties. SIAM J. on Control and Optimization, 49:2494-2517, 2011.

[13] J.F. Bonnans and H. Zidani. Optimal control problems with partially polyhedric constraints. SIAM J. Control Optim., 37(6):1726-1741 (electronic), 1999.

[14] E. Casas. Pontryagin's principle for state-constrained boundary control problems of semilinear parabolic equations. SIAM J. Control and Optimization, 35-4:1297-1237, 1997.

[15] E. Casas and M. Mateos. Second order optimality conditions for semilinear elliptic control problems with finitely many state constraints. SIAM J. Control Optim., 40(5):1431-1454 (electronic), 2002.

[16] E. Casas, J.-P. Raymond, and H. Zidani. Pontryagin principle for local solutions of control problems with mixed state control constraints. SIAM J. Control and Optimization, 39-4:1182$1203,2000$.

[17] E. Casas and F. Tröeltzsch. Second order necessary optimality conditions for some stateconstrained control problems of semilinear elliptic equations. Appl. Math. Optim., 39:211-228, 1999.

[18] E. Casas and F. Tröeltzsch. Second order necessary and sufficient optimality conditions for optimization problems and applications to control theory. SIAM J. Optim., 13:406-431, 2002.

[19] E. Casas and F. Tröeltzsch. Recent advances in the analysis of pointwise state-constrained elliptic optimal control problems. ESAIM Cont. Optim. Calc. Var., 16-3:581-600, 2010.

[20] E. Casas and F. Tröeltzsch. Second order analysis for optimal control problems: Improving results expected from abstract theory. SIAM J. on Optimization, 22(1):261-279, March 2012.

[21] E. Casas, F. Tröeltzsch, and A. Unger. Second order sufficient optimality conditions for a nonlinear elliptic control problem. Journal for Analysis and its Applications, 15:687-707, 1996.

[22] E. Casas, F. Tröeltzsch, and A. Unger. Second order sufficient optimality conditions for some state-constrained control problems of semilinear elliptic equations. SIAM J. Control Optim., 38:369-1391, 2000.

[23] A. V. Dmitruk. Jacobi type conditions for singular extremals. Control and Cybernetics, Vol. 37, no 2:285-306, 2008.

[24] L.C. Evans. Partial differential equations. Amer. Math Soc., Providence, RI, 1998. Graduate Studies in Mathematics 19.

[25] D. Gilbarg and N.S. Trudinger. Elliptic partial differential equations of second order, 2nd edition. Springer Verlag, Berlin, 1983.

[26] H. Goldberg and F. Tröeltzsch. Second order sufficient optimality conditions for a class of non-linear parabolic boundary control problems. SIAM J. Control Optim., 31:1007-1025, 1993.

[27] M. Hinze, R. Pinnau, M. Ulbrich, and S. Ulbrich. Optimization with PDE constraints. Springer, New York, 2008. 
[28] B. Hu and J. Yong. Pontryagin maximum principle for semilinear and quasilinear parabolic equations with pointwise state constraints. SIAM Journal on Control and Optimization, 336:1857-1880, 1995.

[29] A. Ioffe. Necessary and sufficient conditions for a local minimum. 3: Second order conditions and augmented duality. SIAM Journal on Control and Optimization, 17(2):266-288, 1979.

[30] A.D. Ioffe and V.M. Tihomirov. Theory of Extremal Problems. North-Holland Publishing Company, Amsterdam, 1979.

[31] K. Ito and K. Kunisch. Lagrange multiplier approach to variational problems and applications. Advances in design and control. Society for Industrial and Applied Mathematics, 2008.

[32] O. A. Ladyzhenskaya, V.A. Solonnikov, and N.N. Ural'ceva. Linear and quasilinear equations of parabolic type. Translation of Mathematical Monographs, Vol. 23. American Mathematical Society, Providence, R.I., 1967.

[33] J.-L. Lions. Quelques méthodes de resolution de problèmes aux limites nonlinèaires. DunodGauthier-Villars, Paris, 1969.

[34] J.-L. Lions and E. Magenes. Problèmes aux limites non homogènes et applications. Vol. 1. Travaux et Recherches Mathématiques, No. 17. Dunod, Paris, 1968.

[35] K. Malanowski. Sensitivity analysis for parametric optimal control of semilinear parabolic equations. J. Convex Anal., 9(2):543-561, 2002. Special issue on optimization (Montpellier, 2000).

[36] H. Mäurer. First and second order sufficient optimality conditions in mathematical programming and optimal control. Math. Programming Stud., 24:163-177, 1981.

[37] S. M. Robinson. Strongly regular generalized equations. Mathematics of Operations Research, $5(1): 43-62,1980$.

[38] R. T. Rockafellar. Integral functionals, normal integrands and measurable selections. In Nonlinear operators and the calculus of variations (Summer School, Univ. Libre Bruxelles, Brussels, 1975), pages 157-207. Lecture Notes in Math., Vol. 543. Springer, Berlin, 1976.

[39] A. Rösch and F. Tröeltzsch. Sufficient second-order optimality conditions for an elliptic optimal control problem with pointwise control-state constraints. SIAM J. Optim., 17-3:776-794, 2006.

[40] A. Shapiro. Perturbation analysis of optimization problems in Banach spaces. Numer. Funct. Anal. Optim., 13(1-2):97-116, 1992.

[41] F. Tröeltzsch. Optimal Control of Partial Differential Equations - Theory, Methods and Applications. Graduate Studies in Mathematics, Vol. 112. American Mathematical Society, Providence, Rhode Island, 2010. 